\title{
A Unified Analysis of Claim Costs up to Ruin in a Markovian Arrival Risk Model
}

\author{
Eric C.K. Cheung* and Runhuan Feng ${ }^{\dagger}$
}

\begin{abstract}
An insurance risk model where claims follow a Markovian arrival process (MArP) is considered in this paper. It is shown that the expected present value of total operating costs up to default $H$, as a generalization of the classical Gerber-Shiu function, contains more non-trivial quantities than those covered in Cai et al. (2009), such as all moments of the discounted claim costs until ruin. However, it does not appear that the Gerber-Shiu function $\phi$ with a generalized penalty function which additionally depends on the surplus level immediately after the second last claim before ruin (Cheung et al. (2010a)) is contained in $H$. This motivates us to investigate an even more general function $Z$ from which both $H$ and $\phi$ can be retrieved as special cases. Using a matrix version of Dickson-Hipp operator (Feng (2009b)), it is shown that $Z$ satisfies a Markov renewal equation and hence admits a general solution. Applications to other related problems such as the matrix scale function, the minimum and maximum surplus levels before ruin are given as well.
\end{abstract}

Keywords: Claim costs up to ruin; Generalized penalty function; Gerber-Shiu function; Markovian arrival process; Risk model; Dickson-Hipp operator; Markov renewal equation.

\section{Introduction}

\subsection{Generalizations of the Gerber-Shiu function}

In classical ruin theory, the expected present value (EPV) of penalty at ruin, also known as the Gerber-Shiu function (Gerber and Shiu (1998)), has been one of the most popular quantities of interest in recent ten years. For the purpose of comparison with other quantities, we introduce the structure of this function in general terms. Let $\mathcal{U}=\left\{U_{t}\right\}_{t \geq 0}$ be a stochastic process representing the surplus of an insurer under consideration. The process $\mathcal{U}$ is defined on a probability space $\left(\Omega, \mathcal{F},\left\{\mathbb{P}^{u}, u \in \mathbb{R}\right\}\right)$ satisfying the usual conditions, with $\left\{\mathbb{P}^{u}, u \in \mathbb{R}\right\}$ a family of probability measures

\footnotetext{
*Department of Statistics and Actuarial Science, University of Hong Kong, Pokfulam, Hong Kong, eckc@hku.hk.

${ }^{\dagger}$ Department of Mathematics, University of Illinois at Urbana-Champaign, 1409 W. Green Street Urbana, IL 61801, USA, rfeng@illinois.edu.
} 
associated with the process under which $\mathbb{P}^{u}\left(U_{0}=u\right)=1$. The quantity of concern to the insurer is the time of ruin defined as $\tau:=\inf \left\{t \geq 0: U_{t}<0\right\}$. With slight variations under specific models, the now classical Gerber-Shiu function is typically defined as

$$
\phi_{C L}(u):=\mathbb{E}^{u}\left[e^{-\delta \tau} w\left(U_{\tau^{-}},\left|U_{\tau}\right|\right) I(\tau<\infty)\right], \quad u \geq 0
$$

where $\mathbb{E}^{u}$ is the expectation taken under the measure $\mathbb{P}^{u}, \delta \geq 0$ can be interpreted as a Laplace transform argument or the force of interest, $w$ is the so-called 'penalty function', and $I(A)$ is the indicator function for the event $A$. It is well-known that the above function provides a systematic tool to exploit the relationship among the time of ruin $\tau$, surplus prior to ruin $U_{\tau^{-}}$and deficit at ruin $\left|U_{\tau}\right|$.

In the recent literature, there have been various attempts to generalize the Gerber-Shiu function in order to accommodate more quantities of interest. One of such attempts was made by Cheung et al. (2010a) to incorporate an additional variable into the penalty function, namely the surplus level at the time of the penultimate claim prior to ruin. Let $\mathcal{N}=\left\{N_{t}\right\}_{t \geq 0}$ be the number of claims process and $\left\{T_{k}\right\}_{k=1}^{\infty}$ be the sequence of arrival times of insurance claims (which is such that $N_{t}=\sup \left\{k \in \mathbb{N}: T_{k} \leq t\right\}$ with the definition $\left.T_{0}=0\right)$. Then $R_{n}=U_{T_{n}}$ is the surplus level of $\mathcal{U}$ immediately after the $n$-th claim for $n=1,2, \ldots$ with the definition $R_{0}=U_{0}$. The generalization in their work is formulated as

$$
\phi(u):=\mathbb{E}^{u}\left[e^{-\delta \tau} w\left(U_{\tau^{-}},\left|U_{\tau}\right|, R_{N_{\tau}-1}\right) I(\tau<\infty)\right], \quad u \geq 0,
$$

where $w$ is now the extended penalty function. In addition to all that is known to the classical Gerber-Shiu function (1.1), the generalized version (1.2) enables us to analyze the joint distribution of the original three quantities along with the newly introduced $R_{N_{\tau}-1}$. In particular, Cheung et al. (2010a) used $\phi$ to obtain the joint and marginal distributions of the last interclaim time before ruin occurs and the claim causing ruin in the compound Poisson risk model. The analysis of $\phi$ in other risk models can be found in Cheung and Landriault (2009b), Cheung et al. (2010b), Woo (2010, 2012) and Cheung (2011), whereas the application of $\phi$ to derive some ordering properties for various ruin-related quantities was done by Cheung et al. (2011).

Another development was made by Cai et al. (2009) to analyze quantities pertaining to the survival of insurance business from the time of origin to the time of ruin. The quantity of interest is interpreted as the EPV of total operating costs up to ruin, namely

$$
H(u):=\mathbb{E}^{u}\left[\int_{0}^{\tau} e^{-\delta t} l\left(U_{t}\right) \mathrm{d} t\right], \quad u \geq 0,
$$

where the 'cost function' $l(x)$ represents the operating cost depending on the current surplus level $x$ for $x \geq 0$. In the context of the compound Poisson risk model, Cai et al. (2009) showed that the above function encompasses a variety of quantities including the classic Gerber-Shiu function (1.1), EPV of claim costs up to ruin, EPV of insurer's utility, EPV of dividends paid up to ruin, etc. Added to the list as shown in this paper include the moments of discounted claim costs up to ruin and the moments of operating costs up to ruin in a risk model with Markovian claim arrivals as described in Section 1.2. The function $H$ was also studied by Feng (2009a,b) for a renewal risk 
model with phase-type interclaim times and used in Feng (2011) and Feng and Shimizu (2013a,b) to obtain potential measures of jump diffusion processes and Lévy processes.

An imminent question arises with regard to the comparison of the two distinct generalizations (1.2) and (1.3): Do they belong to an even larger class of functions? In this paper, we shall demonstrate that both can indeed be accommodated by a more general form. More precisely, we further allow the cost function $l$ to depend on the surplus level immediately after the previous claim. Then such a more general function is defined as

$$
Z(u):=\mathbb{E}^{u}\left[\int_{0}^{\tau} e^{-\delta t} l\left(U_{t}, U_{T_{N_{t}}}\right) \mathrm{d} t\right], \quad u \geq 0,
$$

with $l$ now being the extended cost function.

\subsection{Markovian arrival process}

In this paper, we assume that the insurer's surplus process $\mathcal{U}=\left\{U_{t}\right\}_{t \geq 0}$ is described by

$$
U_{t}=u+c t-\sum_{k=1}^{N_{t}} Y_{k}, \quad t \geq 0,
$$

where claims arrive according to a Markovian arrival process. The constant $u \geq 0$ is the initial surplus level and $c>0$ is the incoming premium rate per unit time. Moreover, $\mathcal{N}=\left\{N_{t}\right\}_{t \geq 0}$ is the number of claims process and $\mathcal{J}=\left\{J_{t}\right\}_{t \geq 0}$ is a homogeneous irreducible continuous-time Markov chain (CTMC) with finite state space $E=\{1, \ldots, m\}$ and arbitrary initial probability vector. The natural filtration of this bivariate Markov process $(\mathcal{N}, \mathcal{J})=\left\{\left(N_{t}, J_{t}\right)\right\}_{t>0}$ is denoted by $\left\{\mathcal{F}_{t}\right\}_{t>0}$ associated with the family of measures $\left\{\mathbb{P}^{(u, i)}\right\}$ each of which determines $\mathbb{P}^{(u, i)}\left(U_{0}=u, J_{0}=i\right)=1$. The evolution of the bivariate Markov process on the state space $\mathbb{N} \times E$ is governed by the $m \times m$ square matrices $\boldsymbol{\Lambda}^{0}$ and $\boldsymbol{\Lambda}^{1}$. The $(i, j)$-th element of $\boldsymbol{\Lambda}^{0}$, namely $\lambda_{i j}^{0} \geq 0$, is the instantaneous rate of transition of $\mathcal{J}$ from state $i$ to state $j \neq i$ in $E$ without an associated claim occurrence. On the other hand, the $(i, j)$-th element of the matrix $\boldsymbol{\Lambda}^{1}$, namely $\lambda_{i j}^{1} \geq 0$, is the instantaneous rate of transition from state $i$ to state $j$ in $E$ with an accompanying claim. The diagonal elements of $\Lambda^{0}$ are assumed to be negative and such that the sum of the elements on each row of the matrix $\Lambda^{0}+\Lambda^{1}$ is zero. The sequence $\left\{Y_{k}\right\}_{k=1}^{\infty}$ represents insurance claims with $Y_{k}$ being the size of the $k$-th claim. For a transition of the underlying CTMC $\mathcal{J}$ from state $i$ to state $j$ at the time of a claim, the accompanying claim is assumed to have density $p_{i j}(\cdot)$, cumulative distribution function $P_{i j}(\cdot)$,

survival function $\bar{P}_{i j}(\cdot):=1-P_{i j}(\cdot)$ and mean $\mu_{i j}$. For later use it will be convenient to define the matrix $\mathbf{p}(\cdot):=\left[p_{i j}(\cdot)\right]_{i, j=1}^{m}$. The positive security loading condition (under which ruin does not happen with probability 1) is given by (e.g. Cheung and Landriault (2010))

$$
\sum_{i=1}^{m} \pi_{i} \sum_{j=1}^{m} \lambda_{i j}^{1} \mu_{i j}<c
$$

Here $\pi_{i}$ 's are the stationary probabilities of the CTMC $\mathcal{J}$ which are the solution of the system of linear equations comprising

$$
\left\{\begin{array}{l}
\sum_{j=1, j \neq i}^{m} \pi_{j}\left(\lambda_{j i}^{0}+\lambda_{j i}^{1}\right)=-\pi_{i} \lambda_{i i}^{0}, \quad i \in E . \\
\sum_{j=1}^{m} \pi_{j}=1 .
\end{array}\right.
$$


The condition (1.5) may or may not be assumed. More specifically, as a general rule of thumb, we only require either $(1.5)$ or $\delta>0$ (see Remark 4).

Throughout the paper, we shall use the acronym MArP for Markovian arrival process. Readers are referred to Neuts (1989) and Latouche and Ramaswami (1999) for introduction to MArP. Analysis of MArP risk models can be found in e.g. Badescu et al. (2005, 2007a,b), Ahn and Badescu (2007), Ahn et al. (2007), and Cheung and Landriault (2009a, 2010). Well-known special cases of MArP risk models include Sparre Andersen models with phase-type inter-arrival times (e.g. Cheung (2007) and Feng (2009a,b)), the Markov-modulated (or regime-switching) risk processes (e.g. Li and Lu (2007, 2008) and Zhu and Yang $(2008,2009)$ ), and the semi-Markovian risk model by Albrecher and Boxma (2005).

\subsection{Notation and organization of paper}

We shall use the following convention of matrix notation throughout the paper. Whenever $\mathbf{A}$ is a matrix, the $(i, j)$-th element is denoted by $[\mathbf{A}]_{i j}=A_{i j}$ and the $j$-th column is denoted by $\mathbf{A}_{\cdot j}$ by convention. Whenever $\mathbf{A}$ is a vector, the $i$-th entry is denoted by $[\mathbf{A}]_{i}=A_{i}$. All vectors in this paper are column vectors by default. We shall also use the superscript $\top$ to denote the transpose of a matrix. The notation $\circ$ denotes the Hadamard product (entrywise multiplication), i.e. $[\mathbf{A} \circ \mathbf{B}]_{i j}=A_{i j} B_{i j}$ for all $i, j$ 's. The expectation $\mathbb{E}^{(u, i)}$ is taken under the measure $\mathbb{P}^{(u, i)}$, and $\mathbb{E}^{u}$ is treated as a vector with $i$-th element being the expectation $\mathbb{E}^{(u, i)}$. The symbols $\mathbf{0}, \mathbf{I}$ and $\mathbf{1}$ are used for zero matrix, identity matrix and a vector of ones respectively, all of appropriate dimension. Although most quantities of interest in this paper are dependent on the force of interest $\delta$, we shall suppress the argument $\delta$ for notational brevity.

The rest of the paper is organized as follows. In Section 2, various new moment-based quantities and a transition kernel characterizing $\phi$ are shown to be non-trivial special cases of $H$ under different choices of the cost function $l$, although $\phi$ itself does not appear to be contained in $H$. Section 3 unifies the study of all quantities by proposing the novel quantity $Z$ defined via (1.4) which includes not only $H$ but also $\phi$ as special cases. The derivation of the general solution to $Z$ relies heavily on the use of matrix Dickson-Hipp operators. The notion of matrix Dickson-Hipp operators was introduced by Feng (2009b) which serves as an extension of the classical scalar counterpart proposed by Dickson and Hipp (2001). Section 4 is concerned with a few examples to see how the results and techniques involved can be applied to related problems such as the minimum and maximum surplus levels before ruin.

\section{Non-trivial special cases of $H$}

\subsection{Moment-based quantities in relation to discounted costs up to ruin}

We first aim at extending the so-called 'EPV of total claim costs up to ruin' introduced by Cai et al. (2009, Equation (1.2)) (see also Feng (2009a, Equation (4.15) and 2009b, Section 5.2)), which is known to contain the classical Gerber-Shiu function $\phi_{C L}$ as a special case (see Remark 1). In 
particular, we shall consider the higher moments of the discounted total claim costs up to ruin. In the context of the MArP risk model it is defined by, for $n=0,1, \ldots$,

$$
C_{n, i}(u):=\mathbb{E}^{(u, i)}\left[\left(\sum_{k=1}^{N_{\tau}} e^{-\delta T_{k}} \varpi_{\left(J_{T_{k}^{-}}, J_{T_{k}}\right)}\left(U_{T_{k}^{-}}, U_{T_{k}}\right)\right)^{n}\right], \quad u \geq 0
$$

where $\varpi_{i j}$ is a function on $[0, \infty) \times(-\infty, \infty)$ representing the 'cost' associated with a claim if the claim is a result of a transition of $\mathcal{J}$ from state $i$ to state $j$. For this quantity we assume a strictly positive discount factor of $\delta>0$, as the total claim costs may be infinite without discounting when ruin does not occur. Note that $C_{0, i}(u)=1$ by definition, and we define the column vector $\mathbf{C}_{n}(u)$ with $i$-th element $\left[\mathbf{C}_{n}(u)\right]_{i}:=C_{n, i}(u)$.

Remark 1 When $n=1$, it is known from Feng (2009a, Equation (4.28)) that the classical GerberShiu function under a penalty function of $w(\cdot, \cdot)$ is a special case of the EPV of total claim costs up to ruin. More precisely, in the present context the Gerber-Shiu function

$$
\phi_{C L, i j}(u):=\mathbb{E}^{(u, i)}\left[e^{-\delta \tau} w\left(U_{\tau^{-}},\left|U_{\tau}\right|\right) I\left(\tau<\infty, J_{\tau}=j\right)\right], \quad u \geq 0,
$$

can be retrieved from $C_{1, i}(u)$ by letting

$$
\varpi_{k l}(x, y)= \begin{cases}0, & y \geq 0 . \\ w(x,-y) I(l=j), & y<0\end{cases}
$$

An alternative quantity of interest would be, for $n=0,1, \ldots$,

$$
\hat{C}_{n, i j}(u):=\mathbb{E}^{(u, i)}\left[\left(\sum_{k=1}^{N_{\tau}} e^{-\delta T_{k}} \varpi_{\left(J_{T_{k}^{-}}, J_{T_{k}}\right)}\left(U_{T_{k}^{-}}, U_{T_{k}}\right)\right)^{n} I\left(\tau<\infty, J_{\tau}=j\right)\right], \quad u \geq 0 .
$$

The quantity $\hat{C}_{n, i j}$ only takes into account the discounted total claim costs until ruin if ruin occurs while $\mathcal{J}$ is in state $j$. In contrast, the state at ruin is not present in the definition of $C_{n, i}$ in $(2.1)$ allowing the possibility that ruin does not occur (under $(1.5)$ ). When $n=0, \hat{C}_{0, i j}(u)$ reduces to the probability of ruin in state $j$, namely $\psi_{i j}(u):=\mathbb{P}^{(u, i)}\left(\tau<\infty, J_{\tau}=j\right)$, which is a special case of $\phi_{C L, i j}(u)$ in $(2.2)$ by letting $\delta=0$ and $w(\cdot, \cdot) \equiv 1$. Unlike the case of $C_{n, i}$, we allow $\delta=0$ for $\hat{C}_{n, i j}$. One can obtain from (2.3) the moments of the number of claims up to ruin by letting $\delta=0$ and $\varpi_{i j}(\cdot, \cdot) \equiv 1$ for all $i, j$ 's. We also define the square matrices $\hat{\mathbf{C}}_{n}(u)$ and $\boldsymbol{\Psi}(u)$ with $(i, j)$-th elements $\left[\hat{\mathbf{C}}_{n}(u)\right]_{i j}:=\hat{C}_{n, i j}(u)$ and $[\Psi(u)]_{i j}:=\psi_{i j}(u)$ respectively. Note that the moments of discounted total claim costs without ruin occurring can be obtained as

$$
\mathbb{E}^{(u, i)}\left[\left(\sum_{k=1}^{N_{\tau}} e^{-\delta T_{k}} \varpi_{\left(J_{T_{k}^{-}}, J_{T_{k}}\right)}\left(U_{T_{k}^{-}}, U_{T_{k}}\right)\right)^{n} I(\tau=\infty)\right]=C_{n, i}(u)-\sum_{j=1}^{m} \hat{C}_{n, i j}(u), \quad u \geq 0 .
$$

See Badescu and Landriault (2008) for a similar situation in which the dividend moments for MArP risk models with threshold strategies are distinguished according to whether ruin occurs. 
By the same logic, we may also extend the EPV of total operating costs up to ruin defined by (1.3) and consider higher moments defined in the present context by, for $n=0,1, \ldots$,

$$
H_{n, i}(u):=\mathbb{E}^{(u, i)}\left[\left(\int_{0}^{\tau} e^{-\delta t} l_{J_{t}}\left(U_{t}\right) \mathrm{d} t\right)^{n}\right], \quad u \geq 0
$$

where $l_{j}$ is the cost function and is allowed to depend on the state of the Markov chain $\mathcal{J}$. The generalized version (2.4) for $n>1$ allows for the calculation of, for example, the moments of accumulated utility of the insurer. Note that $H_{0, i}(u)=1$, and we define $\left[\mathbf{H}_{n}(u)\right]_{i}:=H_{n, i}(u)$ and $[\mathbf{l}(u)]_{i}:=l_{i}(u)$. Similarly, we can also define the moments of discounted total operating costs up to ruin with ruin occurring by, for $n=0,1, \ldots$,

$$
\hat{H}_{n, i j}(u):=\mathbb{E}^{(u, i)}\left[\left(\int_{0}^{\tau} e^{-\delta t} l_{J_{t}}\left(U_{t}\right) \mathrm{d} t\right)^{n} I\left(\tau<\infty, J_{\tau}=j\right)\right], \quad u \geq 0 .
$$

The function $\hat{H}_{n, i j}$ is different from $H_{n, i}$ in that the operating costs are counted only for the cases where ruin occurs in state $j$. When $n=0, \hat{H}_{0, i j}$ reduces to the probability of ruin $\psi_{i j}$. For convenience we define the matrix $\hat{\mathbf{H}}_{n}(u):=\left[\hat{H}_{n, i j}(u)\right]_{i, j=1}^{m}$.

As we shall see, the above moment-based quantities can be retrieved from $\mathbf{H}_{1}$ under appropriate choices of the cost function 1 . To make the presentation clear, we reinstate the first moment of discounted total operating costs up to ruin with a cost function $\mathbf{l}^{*}$ (such that $\left[\mathbf{l}^{*}(\cdot)\right]_{j}:=l_{j}^{*}(\cdot)$ ) and force of interest $\delta^{*}$ as

$$
\mathbf{H}^{*}(u):=\mathbb{E}^{u}\left[\int_{0}^{\tau} e^{-\delta^{*} t} l_{J_{t}}^{*}\left(U_{t}\right) \mathrm{d} t\right], \quad u \geq 0,
$$

which is the column vector with $i$-th element $\left[\mathbf{H}^{*}(u)\right]_{i}=H_{i}^{*}(u):=\mathbb{E}^{(u, i)}\left[\int_{0}^{\tau} e^{-\delta^{*} t} l_{J_{t}}^{*}\left(U_{t}\right) \mathrm{d} t\right]$.

Proposition 1 Define $\varpi_{k}(\cdot, \cdot):=\left[\varpi_{i j}^{k}(\cdot, \cdot)\right]_{i, j=1}^{m}$. For $n=1,2, \ldots$,

1. $\mathbf{C}_{n}$ is a special case of $\mathbf{H}^{*}$ with $\delta^{*}=n \delta$ and

$$
\mathbf{l}^{*}(x)=\sum_{k=1}^{n-1}\left(\begin{array}{l}
n \\
k
\end{array}\right) \int_{0}^{x}\left(\Lambda^{1} \circ \mathbf{p}(y) \circ \varpi_{n-k}(x, x-y)\right) \mathbf{C}_{k}(x-y) \mathrm{d} y+\int_{0}^{\infty}\left(\boldsymbol{\Lambda}^{1} \circ \mathbf{p}(y) \circ \varpi_{n}(x, x-y)\right) \mathbf{1} \mathrm{d} y .
$$

2. $\hat{\mathbf{C}}_{n, j}$ is a special case of $\mathbf{H}^{*}$ with $\delta^{*}=n \delta$ and

$$
\mathbf{l}^{*}(x)=\sum_{k=0}^{n-1}\left(\begin{array}{l}
n \\
k
\end{array}\right) \int_{0}^{x}\left(\Lambda^{1} \circ \mathbf{p}(y) \circ \varpi_{n-k}(x, x-y)\right) \hat{\mathbf{C}}_{k, j}(x-y) \mathrm{d} y+\int_{x}^{\infty}\left(\Lambda^{1} \circ \mathbf{p}(y) \circ \varpi_{n}(x, x-y)\right)_{\cdot j} \mathrm{~d} y .
$$

Proof. We follow a similar proof as in Feng (2009a, Theorem 4.1). Denote the time of first transition of the underlying Markov chain $\mathcal{J}$ by $\sigma$. Conditioning on $\sigma$ and the resulting claim (if any), we have 


$$
\begin{aligned}
C_{n, i}(u)= & \int_{0}^{\infty} e^{\left(\lambda_{i i}^{0}-n \delta\right) t} \sum_{j=1, j \neq i}^{m} \lambda_{i j}^{0} C_{n, j}(u+c t) \mathrm{d} t \\
& +\int_{0}^{\infty} e^{\left(\lambda_{i i}^{0}-n \delta\right) t} \sum_{j=1}^{m} \lambda_{i j}^{1}\left[\int_{0}^{u+c t} \sum_{k=0}^{n}\left(\begin{array}{l}
n \\
k
\end{array}\right) p_{i j}(y) \varpi_{i j}^{n-k}(u+c t, u+c t-y) C_{k, j}(u+c t-y) \mathrm{d} y\right. \\
& \left.+\int_{u+c t}^{\infty} p_{i j}(y) \varpi_{i j}^{n}(u+c t, u+c t-y) \mathrm{d} y\right] \mathrm{d} t .
\end{aligned}
$$

Note that

$$
\begin{aligned}
& \mathbb{E}^{(u, i)}\left[e^{-n \delta \sigma} C_{n, J_{\sigma}}\left(U_{\sigma}\right)\right] \\
= & \int_{0}^{\infty} e^{\lambda_{i i}^{0} t}\left[\sum_{j=1, j \neq i}^{m} \lambda_{i j}^{0} e^{-n \delta t} C_{n, j}(u+c t)+\sum_{j=1}^{m} \lambda_{i j}^{1} e^{-n \delta t} \int_{0}^{u+c t} p_{i j}(y) C_{n, j}(u+c t-y) \mathrm{d} y\right] \mathrm{d} t,
\end{aligned}
$$

and

$$
\begin{aligned}
& \mathbb{E}^{(u, i)}\left[e^{-n \delta \sigma} \varpi_{\left(J_{\sigma^{-}}, J_{\sigma}\right)}^{n-k}\left(U_{\sigma^{-}}, U_{\sigma}\right) C_{k, J_{\sigma}}\left(U_{\sigma}\right) I\left(T_{1}=\sigma\right)\right] \\
= & \int_{0}^{\infty} e^{\lambda_{i i}^{0} t} \sum_{j=1}^{m} \lambda_{i j}^{1} e^{-n \delta t} \int_{0}^{\infty} p_{i j}(y) \varpi_{i j}^{n-k}(u+c t, u+c t-y) C_{k, j}(u+c t-y) \mathrm{d} y \mathrm{~d} t \\
= & \begin{cases}\int_{0}^{\infty} e^{\lambda_{i i}^{0} t} \sum_{j=1}^{m} \lambda_{i j}^{1} e^{-n \delta t} \int_{0}^{\infty} p_{i j}(y) \varpi_{i j}^{n}(u+c t, u+c t-y) \mathrm{d} y \mathrm{~d} t, & k=0, \\
\int_{0}^{\infty} e^{\lambda_{i i}^{0} t} \sum_{j=1}^{m} \lambda_{i j}^{1} e^{-n \delta t} \int_{0}^{u+c t} p_{i j}(y) \varpi_{i j}^{n-k}(u+c t, u+c t-y) C_{k, j}(u+c t-y) \mathrm{d} y \mathrm{~d} t, & k=1,2, \ldots, n-1,\end{cases}
\end{aligned}
$$

where we follow the usual convention that $C_{0, j}(u) \equiv 1$ for all $u$ and $C_{k, j}(u) \equiv 0$ for $u<0$ and $k=1,2, \ldots$ Therefore, $(2.7)$ can be expressed as

$$
C_{n, i}(u)=\sum_{k=0}^{n-1}\left(\begin{array}{l}
n \\
k
\end{array}\right) \mathbb{E}^{(u, i)}\left[e^{-n \delta \sigma} \varpi_{\left(J_{\sigma^{-}}, J_{\sigma}\right)}^{n-k}\left(U_{\sigma^{-}}, U_{\sigma}\right) C_{k, J_{\sigma}}\left(U_{\sigma}\right) I\left(T_{1}=\sigma\right)\right]+\mathbb{E}^{(u, i)}\left[e^{-n \delta \sigma} C_{n, J_{\sigma}}\left(U_{\sigma}\right)\right] .
$$

Since a claim resulting from a transition in $\mathcal{J}$ from $i$ to $j$ can be viewed as the first event of an independent Poisson random measure $N_{i j}$ with characteristics $\left(\lambda_{i j}^{1}, \nu_{i j}\right)$ where $\mathbb{E}\left[N_{i j}(t, A)\right]:=$ $\lambda_{i j}^{1} t \nu_{i j}(A)$ for all Borel sets $A$ and $\nu_{i j}([0, x])=P_{i j}(x)$, we obtain

$$
\begin{aligned}
& \mathbb{E}^{(u, i)}\left[e^{-n \delta \sigma} \varpi_{\left(J_{\sigma^{-}}, J_{\sigma}\right)}^{n-k}\left(U_{\sigma^{-}}, U_{\sigma}\right) C_{k, J_{\sigma}}\left(U_{\sigma}\right) I\left(T_{1}=\sigma\right)\right] \\
= & \sum_{j=1}^{m} \mathbb{E}^{(u, i)}\left[e^{-n \delta \sigma} \varpi_{\left(J_{\sigma^{-}}, J_{\sigma}\right)}^{n-k}\left(U_{\sigma^{-}}, U_{\sigma}\right) C_{k, J_{\sigma}}\left(U_{\sigma}\right) I\left(T_{1}=\sigma, J_{\sigma}=j\right)\right] \\
= & \sum_{j=1}^{m} \mathbb{E}^{(u, i)}\left[\int_{0}^{\sigma} e^{-n \delta t} \int_{0}^{\infty} \varpi_{i j}^{n-k}\left(U_{t^{-}}, U_{t^{-}}-y\right) C_{k, j}\left(U_{t^{-}}-y\right) N_{i j}(\mathrm{~d} t, \mathrm{~d} y)\right] \\
= & \sum_{j=1}^{m} \mathbb{E}^{(u, i)}\left[\int_{0}^{\sigma} e^{-n \delta t} \int_{0}^{\infty} \varpi_{i j}^{n-k}\left(U_{t^{-}}, U_{t^{-}}-y\right) C_{k, j}\left(U_{t^{-}}-y\right) \lambda_{i j}^{1} p_{i j}(y) \mathrm{d} y \mathrm{~d} t\right]
\end{aligned}
$$




$$
=\sum_{j=1}^{m} \mathbb{E}^{(u, i)}\left[\int_{0}^{\sigma} e^{-n \delta t} \int_{0}^{\infty} \varpi_{i j}^{n-k}\left(U_{t}, U_{t}-y\right) C_{k, j}\left(U_{t}-y\right) \lambda_{i j}^{1} p_{i j}(y) \mathrm{d} y \mathrm{~d} t\right],
$$

with the last equality from the fact that $\left\{t \geq 0: U_{t} \neq U_{t^{-}}\right\}$is a countable set. Hence, we can rewrite (2.8) as

$$
\begin{aligned}
C_{n, i}(u)= & \mathbb{E}^{(u, i)}\left[\int_{0}^{\sigma} e^{-n \delta t} \sum_{k=0}^{n-1}\left(\begin{array}{l}
n \\
k
\end{array}\right) \sum_{j=1}^{m} \int_{0}^{\infty} \varpi_{i j}^{n-k}\left(U_{t}, U_{t}-y\right) C_{k, j}\left(U_{t}-y\right) \lambda_{i j}^{1} p_{i j}(y) \mathrm{d} y \mathrm{~d} t\right] \\
& +\mathbb{E}^{(u, i)}\left[e^{-n \delta \sigma} C_{n, J_{\sigma}}\left(U_{\sigma}\right)\right] .
\end{aligned}
$$

On the other hand, by conditioning on the time of first transition and then applying the strong Markov property to $H_{i}^{*}(u)$, we arrive at

$$
H_{i}^{*}(u)=\mathbb{E}^{(u, i)}\left[\int_{0}^{\sigma} e^{-\delta^{*} t} l_{i}^{*}\left(U_{t}\right) \mathrm{d} t\right]+\mathbb{E}^{(u, i)}\left[e^{-\delta^{*} \sigma} H_{J_{\sigma}}^{*}\left(U_{\sigma}\right)\right],
$$

where it is understood that $H_{i}^{*}(u) \equiv 0$ for $u<0$. It follows immediately by comparing $(2.10)$ and (2.11) that $C_{n, i}(u)$ is a special case of $H_{i}^{*}(u)$ with $\delta^{*}=n \delta$ and

$$
\begin{aligned}
l_{i}^{*}(x) & =\sum_{k=0}^{n-1}\left(\begin{array}{l}
n \\
k
\end{array}\right) \sum_{j=1}^{m} \int_{0}^{\infty} \varpi_{i j}^{n-k}(x, x-y) C_{k, j}(x-y) \lambda_{i j}^{1} p_{i j}(y) \mathrm{d} y \\
& =\sum_{k=1}^{n-1}\left(\begin{array}{l}
n \\
k
\end{array}\right) \sum_{j=1}^{m} \int_{0}^{x} \lambda_{i j}^{1} p_{i j}(y) \varpi_{i j}^{n-k}(x, x-y) C_{k, j}(x-y) \mathrm{d} y+\sum_{j=1}^{m} \int_{0}^{\infty} \lambda_{i j}^{1} p_{i j}(y) \varpi_{i j}^{n}(x, x-y) \mathrm{d} y,
\end{aligned}
$$

whose matrix representation is given by $(2.5)$.

Similarly, for the second statement, we first arrive at

$$
\begin{aligned}
\hat{C}_{n, i j}(u)= & \int_{0}^{\infty} e^{\left(\lambda_{i i}^{0}-n \delta\right) t} \sum_{h=1, h \neq i}^{m} \lambda_{i h}^{0} \hat{C}_{n, h j}(u+c t) \mathrm{d} t \\
+ & \int_{0}^{\infty} e^{\left(\lambda_{i i}^{0}-n \delta\right) t}\left[\sum_{h=1}^{m} \lambda_{i h}^{1} \int_{0}^{u+c t} \sum_{k=0}^{n}\left(\begin{array}{l}
n \\
k
\end{array}\right) p_{i h}(y) \varpi_{i h}^{n-k}(u+c t, u+c t-y) \hat{C}_{k, h j}(u+c t-y) \mathrm{d} y\right. \\
& \left.\quad+\lambda_{i j}^{1} \int_{u+c t}^{\infty} p_{i j}(y) \varpi_{i j}^{n}(u+c t, u+c t-y) \mathrm{d} y\right] \mathrm{d} t .
\end{aligned}
$$

Note that the condition that ruin occurs is already embedded in the renewal argument for the first integral term in the square brackets. Again we have that

$$
\begin{aligned}
& \mathbb{E}^{(u, i)}\left[e^{-n \delta \sigma} \hat{C}_{n,\left(J_{\sigma}, j\right)}\left(U_{\sigma}\right)\right] \\
= & \int_{0}^{\infty} e^{\lambda_{i i}^{0} t}\left[\sum_{h=1, h \neq i}^{m} \lambda_{i h}^{0} e^{-n \delta t} \hat{C}_{n, h j}(u+c t)+\sum_{h=1}^{m} \lambda_{i h}^{1} e^{-n \delta t} \int_{0}^{u+c t} p_{i h}(y) \hat{C}_{n, h j}(u+c t-y) \mathrm{d} y\right] \mathrm{d} t,
\end{aligned}
$$


and

$$
\begin{aligned}
& \mathbb{E}^{(u, i)}\left[e^{-n \delta \sigma} \varpi_{\left(J_{\sigma^{-}}, J_{\sigma}\right)}^{n-k}\left(U_{\sigma^{-}}, U_{\sigma}\right) \hat{C}_{k,\left(J_{\sigma}, j\right)}\left(U_{\sigma}\right) I\left(T_{1}=\sigma\right)\right] \\
= & \left\{\begin{array}{cc}
\int_{0}^{\infty} e^{\lambda_{i i}^{0} t}\left[\sum_{h=1}^{m} \lambda_{i h}^{1} e^{-n \delta t} \int_{0}^{u+c t} p_{i h}(y) \varpi_{i h}^{n}(u+c t, u+c t-y) \hat{C}_{0, h j}(u+c t-y) \mathrm{d} y,\right. \\
\left.\quad+\lambda_{i j}^{1} e^{-n \delta t} \int_{u+c t}^{\infty} p_{i j}(y) \varpi_{i j}^{n}(u+c t, u+c t-y) \mathrm{d} y\right] \mathrm{d} t, & k=0, \\
\int_{0}^{\infty} e^{\lambda_{i i}^{0} t} \sum_{h=1}^{m} \lambda_{i h}^{1} e^{-n \delta t} \int_{0}^{u+c t} p_{i h}(y) \varpi_{i h}^{n-k}(u+c t, u+c t-y) \hat{C}_{k, h j}(u+c t-y) \mathrm{d} y \mathrm{~d} t, & k=1,2, \ldots, n-1,
\end{array}\right.
\end{aligned}
$$

where we follow the usual convention that when $u<0$ one has $\hat{C}_{0, i j}(u)=I(i=j)$ and $\hat{C}_{k, i j}(u) \equiv 0$ for $k=1,2, \ldots$ Now, we can rewrite (2.12) as

$$
\begin{aligned}
\hat{C}_{n, i j}(u)= & \sum_{k=0}^{n-1}\left(\begin{array}{l}
n \\
k
\end{array}\right) \mathbb{E}^{(u, i)}\left[e^{-n \delta \sigma} \varpi_{\left(J_{\sigma}, J_{\sigma}\right)}^{n-k}\left(U_{\sigma^{-}}, U_{\sigma}\right) \hat{C}_{k,\left(J_{\sigma}, j\right)}\left(U_{\sigma}\right) I\left(T_{1}=\sigma\right)\right]+\mathbb{E}^{(u, i)}\left[e^{-n \delta \sigma} \hat{C}_{n,\left(J_{\sigma}, j\right)}\left(U_{\sigma}\right)\right] \\
= & \mathbb{E}^{(u, i)}\left[\int_{0}^{\sigma} e^{-n \delta t} \sum_{k=0}^{n-1}\left(\begin{array}{l}
n \\
k
\end{array}\right) \sum_{h=1}^{m} \int_{0}^{\infty} \varpi_{i h}^{n-k}\left(U_{t}, U_{t}-y\right) \hat{C}_{k, h j}\left(U_{t}-y\right) \lambda_{i h}^{1} p_{i h}(y) \mathrm{d} y \mathrm{~d} t\right] \\
& +\mathbb{E}^{(u, i)}\left[e^{-n \delta \sigma} \hat{C}_{n,\left(J_{\sigma}, j\right)}\left(U_{\sigma}\right)\right]
\end{aligned}
$$

where the last line follows since, as in (2.9),

$$
\begin{aligned}
& \mathbb{E}^{(u, i)}\left[e^{-n \delta \sigma} \varpi_{\left(J_{\sigma^{-}}, J_{\sigma}\right)}^{n-k}\left(U_{\sigma^{-}}, U_{\sigma}\right) \hat{C}_{k,\left(J_{\sigma}, j\right)}\left(U_{\sigma}\right) I\left(T_{1}=\sigma\right)\right] \\
= & \sum_{h=1}^{m} \mathbb{E}^{(u, i)}\left[\int_{0}^{\sigma} e^{-n \delta t} \int_{0}^{\infty} \varpi_{i h}^{n-k}\left(U_{t}, U_{t}-y\right) \hat{C}_{k, h j}\left(U_{t}-y\right) \lambda_{i h}^{1} p_{i h}(y) \mathrm{d} y \mathrm{~d} t\right] .
\end{aligned}
$$

By comparing (2.13) and (2.11), one observes that $\hat{C}_{n, i j}(u)$ is a special case of $H_{i}^{*}(u)$ with $\delta^{*}=n \delta$ and

$$
\begin{aligned}
l_{i}^{*}(x) & =\sum_{k=0}^{n-1}\left(\begin{array}{l}
n \\
k
\end{array}\right) \sum_{h=1}^{m} \int_{0}^{\infty} \varpi_{i h}^{n-k}(x, x-y) \hat{C}_{k, h j}(x-y) \lambda_{i h}^{1} p_{i h}(y) \mathrm{d} y \\
& =\sum_{k=0}^{n-1}\left(\begin{array}{l}
n \\
k
\end{array}\right) \sum_{h=1}^{m} \int_{0}^{x} \lambda_{i h}^{1} p_{i h}(y) \varpi_{i h}^{n-k}(x, x-y) \hat{C}_{k, h j}(x-y) \mathrm{d} y+\int_{x}^{\infty} \lambda_{i j}^{1} p_{i j}(y) \varpi_{i j}^{n}(x, x-y) \mathrm{d} y,
\end{aligned}
$$

which is $(2.6)$.

Proposition 2 For $n=1,2, \ldots$,

1. $\mathbf{H}_{n}$ is a special case of $\mathbf{H}^{*}$ with $\delta^{*}=n \delta$ and $\mathbf{l}^{*}(x)=n \mathbf{l}(x) \circ \mathbf{H}_{n-1}(x)$.

2. $\hat{\mathbf{H}}_{n, \cdot j}$ is a special case of $\mathbf{H}^{*}$ with $\delta^{*}=n \delta$ and $\mathbf{l}^{*}(x)=n \mathbf{l}(x) \circ \hat{\mathbf{H}}_{n-1, \cdot j}(x)$.

Proof. For the first statement, since

$$
\begin{aligned}
n \int_{0}^{T} g(t)\left(\int_{t}^{T} g(s) \mathrm{d} s\right)^{n-1} \mathrm{~d} t & =-n \int_{0}^{T}\left(\int_{t}^{T} g(s) \mathrm{d} s\right)^{n-1} \mathrm{~d}\left(\int_{t}^{T} g(s) \mathrm{d} s\right) \\
& =-\left.n\left(\int_{t}^{T} g(s) \mathrm{d} s\right)^{n}\right|_{0} ^{T}=\left(\int_{0}^{T} g(t) \mathrm{d} t\right)^{n}
\end{aligned}
$$


it follows immediately that

$$
\begin{aligned}
\mathbf{H}_{n}(u) & =\mathbb{E}^{u}\left[n \int_{0}^{\tau} e^{-\delta t} l_{J_{t}}\left(U_{t}\right)\left(\int_{t}^{\tau} e^{-\delta s} l_{J_{s}}\left(U_{s}\right) \mathrm{d} s\right)^{n-1} \mathrm{~d} t\right] \\
& =\mathbb{E}^{u}\left[n \int_{0}^{\infty} e^{-n \delta t} l_{J_{t}}\left(U_{t}\right) I(\tau>t)\left(\int_{t}^{\tau} e^{-\delta(s-t)} l_{J_{s}}\left(U_{s}\right) \mathrm{d} s\right)^{n-1} \mathrm{~d} t\right] \\
& \left.=\mathbb{E}^{u}\left[n \int_{0}^{\infty} e^{-n \delta t} l_{J_{t}}\left(U_{t}\right) I(\tau>t) \mathbb{E}^{u}\left[\left(\int_{t}^{\tau} e^{-\delta(s-t)} l_{J_{s}}\left(U_{s}\right) \mathrm{d} s\right)^{n-1}\right] \mathcal{F}_{t}\right] \mathrm{d} t\right] \\
& =\mathbb{E}^{u}\left[n \int_{0}^{\tau} e^{-n \delta t} l_{J_{t}}\left(U_{t}\right) H_{n-1, J_{t}}\left(U_{t}\right) \mathrm{d} t\right]=\mathbb{E}^{u}\left[\int_{0}^{\tau} e^{-\delta^{*} t} l_{J_{t}}^{*}\left(U_{t}\right) \mathrm{d} t\right],
\end{aligned}
$$

where $\delta^{*}=n \delta$ and $l_{i}^{*}(x)=n l_{i}(x) H_{n-1, i}(x)$. Note that the Tonelli's Theorem and the double expectation formula have been used in the third equality. (More precisely, we are doing operations $\left.\mathbb{E}\left[\int_{0}^{\infty} A(t) \mathrm{d} t\right]=\int_{0}^{\infty} \mathbb{E}[A(t)] \mathrm{d} t=\int_{0}^{\infty} \mathbb{E}\left[\mathbb{E}\left[A(t) \mid \mathcal{F}_{t}\right]\right] \mathrm{d} t=\mathbb{E}\left[\int_{0}^{\infty} \mathbb{E}\left[A(t) \mid \mathcal{F}_{t}\right] \mathrm{d} t\right].\right)$

The proof for the second statement can be carried out in a similar manner as

$$
\begin{aligned}
\hat{\mathbf{H}}_{n, j}(u) & =\mathbb{E}^{u}\left[n \int_{0}^{\tau} e^{-\delta t} l_{J_{t}}\left(U_{t}\right)\left(\int_{t}^{\tau} e^{-\delta s} l_{J_{s}}\left(U_{s}\right) \mathrm{d} s\right)^{n-1} I\left(\tau<\infty, J_{\tau}=j\right) \mathrm{d} t\right] \\
& =\mathbb{E}^{u}\left[n \int_{0}^{\tau} e^{-n \delta t} l_{J_{t}}\left(U_{t}\right) \mathbb{E}^{u}\left[\left(\int_{t}^{\tau} e^{-\delta(s-t)} l_{J_{s}}\left(U_{s}\right) \mathrm{d} s\right)^{n-1} I\left(\tau<\infty, J_{\tau}=j\right) \mid \mathcal{F}_{t}\right] \mathrm{d} t\right] \\
& =\mathbb{E}^{u}\left[n \int_{0}^{\tau} e^{-n \delta t} l_{J_{t}}\left(U_{t}\right) \hat{H}_{n-1,\left(J_{t}, j\right)}\left(U_{t}\right) \mathrm{d} t\right]=\mathbb{E}^{u}\left[\int_{0}^{\tau} e^{-\delta^{*} t} l_{J_{t}}^{*}\left(U_{t}\right) \mathrm{d} t\right]
\end{aligned}
$$

where $\delta^{*}=n \delta$ and $l_{i}^{*}(x)=n l_{i}(x) \hat{H}_{n-1, i j}(x)$.

The implication of Propositions 1 and 2 is that the moment-based quantities $\mathbf{C}_{n}, \hat{\mathbf{C}}_{n, \cdot j}, \mathbf{H}_{n}$ and $\hat{\mathbf{H}}_{n, j}$ can all be studied via $\mathbf{H}_{1}$, and the EPV of total operating costs up to ruin $\mathbf{H}_{1}$ is indeed far more general than the examples listed in Cai et al. (2009). The procedure is recursive as the order of moment $n$ increases. In Proposition 1 the recursion for $\mathbf{C}_{n}$ starts itself at $n=1$, whereas the starting point of the recursion for $\hat{\mathbf{C}}_{n, j}$ is given by $\hat{\mathbf{C}}_{0, \cdot j} \equiv \boldsymbol{\Psi}_{\cdot j}$. In Proposition 2, the starting points are $\mathbf{H}_{0} \equiv \mathbf{1}$ and $\hat{\mathbf{H}}_{0, \cdot j} \equiv \boldsymbol{\Psi}_{\cdot j}$ respectively.

Similarly, we can show that every column vector of $\hat{\mathbf{C}}_{1}$ is a special case of $\mathbf{C}_{1}$. To avoid the ambiguity on the notation of cost functions, we reiterate the definition of $\mathbf{C}^{*}$ with the claim cost function $\varpi^{*}(\cdot, \cdot):=\left[\varpi_{i j}^{*}(\cdot, \cdot)\right]_{i, j=1}^{m}$ as

$$
\mathbf{C}^{*}(u):=\mathbb{E}^{u}\left[\sum_{k=1}^{N_{\tau}} e^{-\delta T_{k}} \varpi_{\left(J_{T_{k}^{-}}, J_{T_{k}}\right)}^{*}\left(U_{T_{k}^{-}}, U_{T_{k}}\right)\right], \quad u \geq 0
$$

which has $i$-th element $\left[\mathbf{C}^{*}(u)\right]_{i}=C_{i}^{*}(u):=\mathbb{E}^{(u, i)}\left[\sum_{k=1}^{N_{\tau}} e^{-\delta T_{k}} \varpi_{\left(J_{T_{k}^{-}}, J_{T_{k}}\right)}^{*}\left(U_{T_{k}^{-}}, U_{T_{k}}\right)\right]$. 
Proposition 3 For $u \geq 0, \hat{\mathbf{C}}_{1, j}(u)$ is a special case of $\mathbf{C}^{*}(u)$ with the claim cost

$$
\varpi_{i k}^{*}(x, y)=\varpi_{i k}(x, y) \psi_{k j}(y),
$$

where it is understood that $\psi_{i j}(y)=I(i=j)$ for $y<0$.

Proof. Note that

$$
\begin{aligned}
\hat{C}_{1, i j}(u) & =\mathbb{E}^{(u, i)}\left[\sum_{k=1}^{N_{\tau}} e^{-\delta T_{k}} \varpi_{\left(J_{T_{k}^{-}}, J_{T_{k}}\right)}\left(U_{T_{k}^{-}}, U_{T_{k}}\right) I\left(\tau<\infty, J_{\tau}=j\right)\right] \\
& =\mathbb{E}^{(u, i)}\left[\sum_{k=1}^{\infty} e^{-\delta T_{k}} \varpi_{\left(J_{T_{k}^{-}}, J_{T_{k}}\right)}\left(U_{T_{k}^{-}}, U_{T_{k}}\right) I\left(\tau \geq T_{k}\right) I\left(\tau<\infty, J_{\tau}=j\right)\right] \\
& =\mathbb{E}^{(u, i)}\left[\sum_{k=1}^{\infty} \mathbb{E}^{(u, i)}\left[e^{-\delta T_{k}} \varpi_{\left(J_{T_{k}^{-}}, J_{T_{k}}\right)}\left(U_{T_{k}^{-}}, U_{T_{k}}\right) I\left(\tau \geq T_{k}\right) I\left(\tau<\infty, J_{\tau}=j\right) \mid \mathcal{F}_{T_{k}}\right]\right] \\
& =\mathbb{E}^{(u, i)}\left[\sum_{k=1}^{\infty} e^{-\delta T_{k}} \varpi_{\left(J_{T_{k}^{-}}, J_{T_{k}}\right)}\left(U_{T_{k}^{-}}, U_{T_{k}}\right) I\left(\tau \geq T_{k}\right) \mathbb{E}^{\left(U_{T_{k}}, J_{T_{k}}\right)}\left[I\left(\tau<\infty, J_{\tau}=j\right)\right]\right] \\
& =\mathbb{E}^{(u, i)}\left[\sum_{k=1}^{N_{\tau}} e^{-\delta T_{k}} \varpi_{\left(J_{T_{k}^{-}}, J_{T_{k}}\right)}\left(U_{T_{k}^{-}}, U_{T_{k}}\right) \psi_{\left(J_{T_{k}}, j\right)}\left(U_{T_{k}}\right)\right] \\
& =\mathbb{E}^{(u, i)}\left[\sum_{k=1}^{N_{\tau}-1} e^{-\delta T_{k}} \varpi_{\left(J_{T_{k}^{-}}, J_{T_{k}}\right)}\left(U_{T_{k}^{-}}, U_{T_{k}}\right) \psi_{\left(J_{T_{k}}, j\right)}\left(U_{T_{k}}\right)+e^{-\delta \tau} \varpi_{\left(J_{\tau^{-}}, J_{\tau}\right)}\left(U_{\tau^{-}}, U_{\tau}\right) I\left(J_{\tau}=j\right)\right] .
\end{aligned}
$$

In the third equality the double expectation formula for filtration at random time is used (Karatzas and Shreve (1991, Problem 2.17)). Thus, $\hat{C}_{1, i j}(u)$ is a special case of $C_{i}^{*}(u)$ with the claim cost function $\varpi_{i k}^{*}$ as in (2.15).

Proposition 3 is quite intuitive: (while ruin has not occurred) the claim cost associated with a claim is included in $\hat{C}_{1, i j}(u)$ if and only if ruin occurs in state $j$ in the future.

\subsection{A transition kernel $\chi$ characterizing $\phi$}

Next, we are interested in a quantity introduced by Cheung (2011) that is known to play a crucial role in analyzing the Gerber-Shiu function (1.2) in the context of a dependent Sparre Andersen risk model. It is interpreted as the discounted kernel density of reaching a certain surplus level after an arbitrary number of claims prior to ruin. In the present risk model with Markovian arrivals, it has a matrix counterpart which is defined as $\boldsymbol{\chi}(u, z):=\left[\chi_{i j}(u, z)\right]_{i, j=1}^{m}$ with

$$
\chi_{i j}(u, z):=\sum_{k=1}^{\infty} \mathbb{E}^{(u, i)}\left[e^{-\delta T_{k}} \Delta\left(U_{T_{k}}-z\right) I\left(T_{k}<\tau, J_{T_{k}}=j\right)\right], \quad u, z \geq 0 .
$$

Here $\Delta$ represents the Dirac delta function which assigns a probability mass 1 to the point 0 . 
Proposition $4 \chi_{\cdot j}(u, z)$ is a special case of $\mathbf{H}^{*}(u)$ with $\delta^{*}=\delta$ and

$$
\mathbf{l}^{*}(x)= \begin{cases}\left(\mathbf{\Lambda}^{1} \circ \mathbf{p}(x-z)\right) \cdot j, & z<x . \\ 0, & z \geq x .\end{cases}
$$

Proof. Because $\left\{T_{k}<\tau\right\}=\left\{k<N_{\tau}\right\}$, we must have

$$
\chi_{i j}(u, z)=\mathbb{E}^{(u, i)}\left[\sum_{k=1}^{N_{\tau}-1} e^{-\delta T_{k}} \Delta\left(U_{T_{k}}-z\right) I\left(J_{T_{k}}=j\right)\right],
$$

which is clearly a special case of $C_{i}^{*}(u)$ (see (2.14)) with the claim cost given by

$$
\varpi_{k l}^{*}(x, y)=\Delta(y-z) I(l=j) .
$$

According to Proposition 1(1) (with $n=1$ ), this $C_{i}^{*}(u)$ is in turn a special case of $H_{i}^{*}(u)$ with $\delta^{*}=\delta$ and

$$
\begin{aligned}
l_{k}^{*}(x) & =\sum_{l=1}^{m} \int_{0}^{\infty} \lambda_{k l}^{1} p_{k l}(y) \varpi_{k l}^{*}(x, x-y) \mathrm{d} y=\sum_{l=1}^{m} \int_{0}^{\infty} \lambda_{k l}^{1} p_{k l}(y) \Delta(x-y-z) I(l=j) \mathrm{d} y \\
& =\lambda_{k j}^{1} p_{k j}(x-z) I(x-z>0) .
\end{aligned}
$$

Hence the result follows.

In the present MArP risk model, the Gerber-Shiu function (1.2) with a generalized penalty is defined as

$$
\phi_{i j}(u):=\mathbb{E}^{(u, i)}\left[e^{-\delta \tau} w\left(U_{\tau^{-}},\left|U_{\tau}\right|, R_{N_{\tau}-1}\right) I\left(\tau<\infty, J_{\tau}=j\right)\right], \quad u \geq 0 .
$$

Its matrix version $\boldsymbol{\Phi}(u):=\left[\phi_{i j}(u)\right]_{i, j=1}^{m}$ is characterized by $\boldsymbol{\chi}$ via the following Proposition.

Proposition 5 The Gerber-Shiu function $\mathbf{\Phi}$ admits the representation

$$
\boldsymbol{\Phi}(u)=\boldsymbol{\beta}(u)+\int_{0}^{\infty} \chi(u, z) \boldsymbol{\beta}(z) \mathrm{d} z, \quad u \geq 0,
$$

where

$$
\boldsymbol{\beta}(u)=\left[\beta_{i j}(u)\right]_{i, j=1}^{m}:=\frac{1}{c} \int_{0}^{\infty} \int_{u}^{\infty} w(x, y, u) e^{\left(-\delta \mathbf{I}+\mathbf{\Lambda}^{0}\right)\left(\frac{x-u}{c}\right)}\left(\boldsymbol{\Lambda}^{1} \circ \mathbf{p}(x+y)\right) \mathrm{d} x \mathrm{~d} y, \quad u \geq 0 .
$$

Proof. First we want to argue that $\boldsymbol{\beta}(u)$ represents the contribution to $\boldsymbol{\Phi}(u)$ by ruin occurring upon the first claim. Note that the probability that the CTMC $\mathcal{J}$ (starting in state $i$ at time 0) is in state $k$ at time $t$ without a claim in the interim is given by $\left[e^{\mathbf{\Lambda}^{0} t}\right]_{i k}$. Being in state $k$ at any instant, a claim that brings $\mathcal{J}$ to state $j$ occurs at rate $\lambda_{k j}^{1}=\left[\boldsymbol{\Lambda}^{1}\right]_{k j}$, and the resulting claim follows density $p_{k j}(\cdot)=[\mathbf{p}(\cdot)]_{k j}$. See e.g. Asmussen (2003, Proposition XI.1.5). Hence, further using the fact that 
$N_{\tau}=1$ (or $\tau=T_{1}$ ) implies $U_{\tau^{-}}=U_{0}+c T_{1}, U_{\tau}=U_{0}+c T_{1}-Y_{1}$ and $R_{N_{\tau}-1}=U_{0}$, the contribution by ruin on the first claim is given by

$$
\begin{aligned}
& \mathbb{E}^{(u, i)}\left[e^{-\delta \tau} w\left(U_{\tau^{-}},\left|U_{\tau}\right|, R_{N_{\tau}-1}\right) I\left(\tau<\infty, J_{\tau}=j, N_{\tau}=1\right)\right] \\
= & \sum_{k=1}^{m} \int_{0}^{\infty} \int_{u+c t}^{\infty} e^{-\delta t} w(u+c t, y-(u+c t), u)\left[e^{\mathbf{\Lambda}^{0} t}\right]_{i k} \lambda_{k j}^{1} p_{k j}(y) \mathrm{d} y \mathrm{~d} t \\
= & \int_{0}^{\infty} \int_{u+c t}^{\infty} e^{-\delta t} w(u+c t, y-(u+c t), u)\left[e^{\mathbf{\Lambda}^{0} t}\left(\boldsymbol{\Lambda}^{1} \circ \mathbf{p}(y)\right)\right]_{i j} \mathrm{~d} y \mathrm{~d} t \\
= & \frac{1}{c} \int_{0}^{\infty} \int_{u}^{\infty} w(x, y, u)\left[e^{\left(-\delta \mathbf{I}+\mathbf{\Lambda}^{0}\right)\left(\frac{x-u}{c}\right)}\left(\boldsymbol{\Lambda}^{1} \circ \mathbf{p}(x+y)\right)\right]_{i j} \mathrm{~d} x \mathrm{~d} y, \quad u \geq 0,
\end{aligned}
$$

where the last line follows from a change of variables. By comparing with (2.19), it is immediate that

$$
\beta_{i j}(u)=\mathbb{E}^{(u, i)}\left[e^{-\delta \tau} w\left(U_{\tau^{-}},\left|U_{\tau}\right|, R_{N_{\tau}-1}\right) I\left(\tau<\infty, J_{\tau}=j, N_{\tau}=1\right)\right], \quad u \geq 0 .
$$

Next, we shall follow similar probabilistic arguments as in Cheung and Landriault (2009b, Section $4.2)$ to prove the representation (2.18) for $\boldsymbol{\Phi}$. Since $\boldsymbol{\beta}(u)$ is the contribution to $\boldsymbol{\Phi}(u)$ by ruin occurring upon the first claim, it remains to prove that the integral term $\int_{0}^{\infty} \boldsymbol{\chi}(u, z) \boldsymbol{\beta}(z) \mathrm{d} z$ is the contribution by ruin on claims subsequent to the first. From (2.16), one observes that $\chi_{i k}(u, z)$ represents the discounted kernel density that the surplus process $\mathcal{U}$ (starting at $U_{0}=u$ and $J_{0}=i$ ) is at level $z$ in state $k$ after an arbitrary number $(\geq 1)$ of claims without ruin in the interim. Starting at this newly established level $z$ in state $k$, the process $\mathcal{U}$ restarts because of its Markovian nature, and if the next claim causes ruin in state $j$, then the surplus level immediately after the penultimate claim prior to ruin $R_{N_{\tau}-1}$ will be precisely $z$, resulting in a contribution of $[\boldsymbol{\beta}(z)]_{k j}$. Because the level $z$ and the state $k$ are arbitrary, summing over $k$ and integrating over $z$ yields

$$
\mathbb{E}^{(u, i)}\left[e^{-\delta \tau} w\left(U_{\tau^{-}},\left|U_{\tau}\right|, R_{N_{\tau}-1}\right) I\left(\tau<\infty, J_{\tau}=j, N_{\tau}>1\right)\right]=\sum_{k=1}^{m} \int_{0}^{\infty} \chi_{i k}(u, z) \beta_{k j}(z) \mathrm{d} z .
$$

By adding (2.20) and (2.21), one obtains $\phi_{i j}(u)$ according to the definition (2.17) and hence the representation (2.18) is proved.

We also refer interested readers to Cheung (2011, Lemma 1 and Proposition 1) for the mathematical details in the scalar case regarding the Proof of (2.18). As discussed in Cheung (2011), the advantage of the representation $(2.18)$ is that the dependence of $\boldsymbol{\Phi}$ on the penalty function $w(\cdot, \cdot, \cdot)$ only appears through the function $\boldsymbol{\beta}$, which is explicitly given by (2.19). Therefore, the GerberShiu function $\boldsymbol{\Phi}$ is essentially characterized by the transition function $\chi$ defined by (2.16), which is independent of the choice of $w(\cdot, \cdot, \cdot)$.

However, although $\chi_{\cdot j}$ characterizing $\boldsymbol{\Phi}$ is a special case of $\mathbf{H}_{1}$, the column vectors of the GerberShiu function $\boldsymbol{\Phi}$ do not appear to be contained in $\mathbf{H}_{1}$. Therefore in the next section we shall study (the matrix version of) $Z$ (see (1.4)) in attempt to unify all quantities in this paper. 


\section{A more general function $Z$}

\subsection{Definition and its special cases}

In the MArP risk model, the matrix counterpart of (1.4) is

$$
\mathbf{Z}(u):=\mathbb{E}^{u}\left[\int_{0}^{\tau} e^{-\delta t} l_{J_{t}}\left(U_{t}, U_{T_{N_{t}}}\right) \mathrm{d} t\right], \quad u \geq 0
$$

with $l_{j}(\cdot, \cdot)$ being the extended cost function which possibly depends on the state of the Markov chain $\mathcal{J}$. In consistence with previous notation, $\mathbf{Z}(u)$ is the column vector with $i$-th element $[\mathbf{Z}(u)]_{i}=$ $Z_{i}(u):=\mathbb{E}^{(u, i)}\left[\int_{0}^{\tau} e^{-\delta t} l_{J_{t}}\left(U_{t}, U_{T_{N_{t}}}\right) \mathrm{d} t\right]$. Obviously, if $l_{j}(\cdot, \cdot)$ does not depend on the second argument, then $\mathbf{Z}$ reduces to $\mathbf{H}_{1}$ which is known to contain $\mathbf{C}_{n}, \hat{\mathbf{C}}_{n, j}, \mathbf{H}_{n}$ and $\hat{\mathbf{H}}_{n, j}$ and $\boldsymbol{\chi}_{\cdot j}$ as special cases from Section 2. For later use we define the column vector of cost functions $\mathbf{l}(\cdot, \cdot)$ such that $[\mathbf{l}(\cdot, \cdot)]_{j}:=l_{j}(\cdot, \cdot)$.

Next we shall focus on two quantities which do not appear to be special cases of $\mathbf{H}_{1}$ but can be retrieved from the extended version $\mathbf{Z}$. The first one can be regarded as an extension of $\mathbf{C}_{1}$ (see (2.1)) and is defined by the column vector $\mathbf{K}(u)$ with $i$-th element $[\mathbf{K}(u)]_{i}:=K_{i}(u)$ given by

$$
K_{i}(u):=\mathbb{E}^{(u, i)}\left[\sum_{k=1}^{N_{\tau}} e^{-\delta T_{k}} \varpi_{\left(J_{T_{k}^{-}}, J_{T_{k}}\right)}\left(U_{T_{k}^{-}}, U_{T_{k}}, U_{T_{k-1}}\right)\right], \quad u \geq 0 .
$$

Here the 'cost' $\varpi_{i j}(\cdot, \cdot, \cdot)$ associated with the $k$-th claim is extended to three arguments and allowed to further depend on the surplus level $U_{T_{k-1}}$ immediately after the previous claim (with $T_{0}=0$ ). Also define the matrix $\varpi(\cdot, \cdot, \cdot):=\left[\varpi_{i j}(\cdot, \cdot, \cdot)\right]_{i, j=1}^{m}$. The following proposition gives the relationship between $\mathbf{K}$ and $\mathbf{Z}$.

Proposition $6 \mathbf{K}$ is a special case of $\mathbf{Z}$ with the cost function

$$
\mathbf{l}(x, z)=\int_{0}^{\infty}\left(\Lambda^{1} \circ \mathbf{p}(y) \circ \varpi(x, x-y, z)\right) \mathbf{1} \mathrm{d} y .
$$

Proof. Following similar arguments as in the Proof of Proposition 5 regarding the use of the matrix exponential $e^{\boldsymbol{\Lambda}^{0} t}$ and the rate matrix $\boldsymbol{\Lambda}^{1}$, conditioning on the time and the resulting amount of the first claim leads to

$$
\begin{aligned}
& \mathbf{K}(u)=\int_{0}^{\infty} e^{-\delta t} e^{\mathbf{\Lambda}^{0} t}\left[\int_{0}^{u+c t}\left(\boldsymbol{\Lambda}^{1} \circ \mathbf{p}(y)\right) \mathbf{K}(u+c t-y) \mathrm{d} y\right. \\
&\left.+\int_{0}^{\infty}\left(\boldsymbol{\Lambda}^{1} \circ \mathbf{p}(y)\right) \circ \varpi(u+c t, u+c t-y, u) \mathbf{1} \mathrm{d} y\right] \mathrm{d} t .
\end{aligned}
$$

For the quantity $\mathbf{Z}(u)$, we break down its contributions at the time of the first claim according to two sources: (1) the present value of future costs given that the process $\mathcal{J}$ restarts at the surplus 
level immediately after the first claim (if it does not cause ruin), and (2) the accumulated value of costs up to the time of the first claim. Therefore,

$$
\mathbf{Z}(u)=\int_{0}^{\infty} e^{-\delta t} e^{\mathbf{\Lambda}^{0} t} \int_{0}^{u+c t}\left(\boldsymbol{\Lambda}^{1} \circ \mathbf{p}(y)\right) \mathbf{Z}(u+c t-y) \mathrm{d} y \mathrm{~d} t+\int_{0}^{\infty} e^{-\delta t} e^{\boldsymbol{\Lambda}^{0} t} \mathbf{l}(u+c t, u) \mathrm{d} t .
$$

Note that in the second integral, the 'current payment method' in the context of life contingencies has been used. A comparison of (3.3) and (3.4) yields the desired result of (3.2).

Another quantity which is a special case of $\mathbf{Z}$ is (each column of) the Gerber-Shiu function $\boldsymbol{\Phi}$ with a generalized penalty function, as shown in the next proposition.

Proposition $7 \boldsymbol{\Phi}_{\cdot j}$ is a special case of $\mathbf{Z}$ with cost function

$$
\mathbf{l}(x, z)=\int_{x}^{\infty} w(x, y-x, z)\left(\Lambda^{1} \circ \mathbf{p}(y)\right)_{\cdot j} \mathrm{~d} y .
$$

Proof. From (2.17) and (3.1), it is clear that $\phi_{i j}$ can be retrieved from $K_{i}$ by choosing

$$
\varpi_{k l}(x, y, z)= \begin{cases}0, & y \geq 0 . \\ w(x,-y, z) I(l=j), & y<0 .\end{cases}
$$

(This actually complements Remark 1.) Hence by Proposition $6, \phi_{i j}$ is a special case of $Z_{i}$ with cost function

$$
l_{k}(x, z)=\sum_{l=1}^{m} \lambda_{k l}^{1} \int_{0}^{\infty} \varpi_{k l}(x, x-y, z) p_{k l}(y) \mathrm{d} y=\lambda_{k j}^{1} \int_{x}^{\infty} w(x, y-x, z) p_{k j}(y) \mathrm{d} y,
$$

which is the desired result.

\subsection{General solution}

In order to derive the general solution to the function $\mathbf{Z}$, we shall apply a matrix operator approach. An operator that is essential to our analysis is a matrix version of the Dickson-Hipp operator introduced by Feng (2009b, Section 2), which is defined as

$$
\mathcal{T}_{\mathbf{S}} \mathbf{h}(x):=e^{\mathbf{S} x} \int_{x}^{\infty} e^{-\mathbf{S} y} \mathbf{h}(y) \mathrm{d} y, \quad x \geq 0,
$$

where $\mathbf{S}$ is a square matrix and $\mathbf{h}$ is a matrix function such that the integral exists. Note that if $\mathbf{S}=s$ is a scalar, then one retrieves the usual Dickson-Hipp operator (see Dickson and Hipp (2001)) applied to the matrix function $\mathbf{h}$, which is understood to be the matrix containing the scalar Dickson-Hipp transforms of the elements of $\mathbf{h}$. If $x=0$ in the above definition, then one can conveniently extend the usual notation of Laplace transforms to matrix quantities and write

$$
\mathcal{L}\{\mathbf{h}\}(\mathbf{S}):=\mathcal{T}_{\mathbf{S}} \mathbf{h}(0)=\int_{0}^{\infty} e^{-\mathbf{S} y} \mathbf{h}(y) \mathrm{d} y .
$$

Therefore one may use the notations $\mathcal{L}\{\mathbf{h}\}(\mathbf{S})$ and $\mathcal{T}_{\mathbf{S}} \mathbf{h}(0)$ interchangeably. We will require the following Lemma in our analysis, and its proof can be found in Feng (2009b). 
Lemma 1 For any square matrices $\mathbf{S}_{1}$ and $\mathbf{S}_{2}$ of the same dimension and a matrix function $\mathbf{h}$,

$$
\mathcal{T}_{\mathbf{S}_{1}}\left(\mathbf{S}_{2}-\mathbf{S}_{1}\right) \mathcal{T}_{\mathbf{S}_{2}} \mathbf{h}(x)=\mathcal{T}_{\mathbf{S}_{1}} \mathbf{h}(x)-\mathcal{T}_{\mathbf{S}_{2}} \mathbf{h}(x), \quad x \geq 0
$$

The following Corollary is a direct consequence of the above Lemma when $x=0$.

Corollary 1 For any square matrices $\mathbf{S}_{1}$ and $\mathbf{S}_{2}$ of the same dimension and a matrix function $\mathbf{h}$,

$$
\mathcal{L}\left\{\left(\mathbf{S}_{2}-\mathbf{S}_{1}\right) \mathcal{T}_{\mathbf{S}_{2}} \mathbf{h}\right\}\left(\mathbf{S}_{1}\right)=\mathcal{L}\{\mathbf{h}\}\left(\mathbf{S}_{1}\right)-\mathcal{L}\{\mathbf{h}\}\left(\mathbf{S}_{2}\right) .
$$

Apart from matrix Dickson-Hipp operators, the convolution operator $*$ will also be used, and it is defined as

$$
\left(\mathbf{h}_{1} * \mathbf{h}_{2}\right)(x):=\int_{0}^{x} \mathbf{h}_{1}(x-y) \mathbf{h}_{2}(y) \mathrm{d} y, \quad x \geq 0,
$$

for two conformable matrix functions $\mathbf{h}_{1}$ and $\mathbf{h}_{2}$. The $n$-fold convolution of a square matrix function $\mathbf{h}$ can then be defined recursively via $\mathbf{h}^{* n}=\left(\mathbf{h}^{*(n-1)} * \mathbf{h}\right)=\left(\mathbf{h} * \mathbf{h}^{*(n-1)}\right)$ for $n \geq 2$ with $\mathbf{h}^{* 1} \equiv \mathbf{h}$. We have the following Lemma for the matrix Dickson-Hipp transform of a convolution.

Lemma 2 For a square matrix $\mathbf{S}$ and two matrix functions $\mathbf{h}_{1}$ and $\mathbf{h}_{2}$,

$$
\mathcal{T}_{\mathbf{S}}\left(\mathbf{h}_{1} * \mathbf{h}_{2}\right)(x)=\left\{\left(\mathcal{T}_{\mathbf{S}} \mathbf{h}_{1}\right) * \mathbf{h}_{2}\right\}(x)+\mathcal{T}_{\mathbf{S}}\left(\mathcal{L}\left\{\mathbf{h}_{1}\right\}(\mathbf{S}) \mathbf{h}_{2}\right)(x), \quad x \geq 0 .
$$

Proof. By the definitions of Dickson-Hipp operator and convolution, one has

$$
\begin{aligned}
\mathcal{T}_{\mathbf{S}}\left(\mathbf{h}_{1} * \mathbf{h}_{2}\right)(x)= & e^{\mathbf{S} x} \int_{x}^{\infty} e^{-\mathbf{S} y} \int_{0}^{y} \mathbf{h}_{1}(y-v) \mathbf{h}_{2}(v) \mathrm{d} v \mathrm{~d} y \\
= & e^{\mathbf{S} x}\left(\int_{0}^{x} \int_{x}^{\infty}+\int_{x}^{\infty} \int_{v}^{\infty}\right) e^{-\mathbf{S} y} \mathbf{h}_{1}(y-v) \mathbf{h}_{2}(v) \mathrm{d} y \mathrm{~d} v \\
= & \int_{0}^{x}\left(e^{\mathbf{S}(x-v)} \int_{x-v}^{\infty} e^{-\mathbf{S} y} \mathbf{h}_{1}(y) \mathrm{d} y\right) \mathbf{h}_{2}(v) \mathrm{d} v \\
& +e^{\mathbf{S} x} \int_{x}^{\infty} e^{-\mathbf{S} v}\left(\int_{v}^{\infty} e^{-\mathbf{S}(y-v)} \mathbf{h}_{1}(y-v) \mathrm{d} y\right) \mathbf{h}_{2}(v) \mathrm{d} v \\
= & \int_{0}^{x}\left[\mathcal{T}_{\mathbf{S}} \mathbf{h}_{1}(x-v)\right] \mathbf{h}_{2}(v) \mathrm{d} v+e^{\mathbf{S} x} \int_{x}^{\infty} e^{-\mathbf{S} v}\left[\mathcal{L}\left\{\mathbf{h}_{1}\right\}(\mathbf{S})\right] \mathbf{h}_{2}(v) \mathrm{d} v \\
= & \left\{\left(\mathcal{T}_{\mathbf{S}} \mathbf{h}_{1}\right) * \mathbf{h}_{2}\right\}(x)+\mathcal{T}_{\mathbf{S}}\left(\mathcal{L}\left\{\mathbf{h}_{1}\right\}(\mathbf{S}) \mathbf{h}_{2}\right)(x),
\end{aligned}
$$

which is the desired result. Note that in the second term above, the Dickson-Hipp operator is only applied to the function $\mathbf{h}_{2}$, i.e. the term $\mathcal{L}\left\{\mathbf{h}_{1}\right\}(\mathbf{S})$ is viewed as a constant.

A useful special case of Lemma 2 is the case $x=0$ in which the first term in (3.6) vanishes, leading to the following Corollary.

Corollary 2 For a square matrix $\mathbf{S}$ and two matrix functions $\mathbf{h}_{1}$ and $\mathbf{h}_{2}$,

$$
\mathcal{L}\left\{\mathbf{h}_{1} * \mathbf{h}_{2}\right\}(\mathbf{S})=\mathcal{L}\left\{\mathcal{L}\left\{\mathbf{h}_{1}\right\}(\mathbf{S}) \mathbf{h}_{2}\right\}(\mathbf{S}) .
$$


As we shall see, the general solution to the matrix function $\mathbf{Z}$ hinges on a crucial matrix $\mathbf{R}$ which is a solution to the equation

$$
\mathcal{L}\left\{\boldsymbol{\Lambda}^{1} \circ \mathbf{p}\right\}(\mathbf{R})=\delta \mathbf{I}-\boldsymbol{\Lambda}^{0}-c \mathbf{R} .
$$

The above equation can be regarded as a matrix extension of Lundberg's fundamental equation in the classical compound Poisson risk model which is the scalar version of the present MArP model. The determination of the matrix $\mathbf{R}$ relies on the more well-known version of Lundberg's fundamental equation

$$
\operatorname{det}\left[\left(\xi-\frac{\delta}{c}\right) \mathbf{I}+\frac{1}{c} \boldsymbol{\Lambda}^{0}+\frac{1}{c} \mathcal{L}\left\{\boldsymbol{\Lambda}^{1} \circ \mathbf{p}\right\}(\xi)\right]=0
$$

which is known to have $m$ roots with non-negative real parts (see e.g. Badescu (2008)). This will be discussed in the Appendix. The following theorem gives the general solution to $\mathbf{Z}$.

Theorem 1 The function $\mathbf{Z}$ satisfies the Markov renewal equation

$$
\mathbf{Z}(u)=\int_{0}^{u} \mathbf{f}(y) \mathbf{Z}(u-y) \mathrm{d} y+\mathbf{v}(u), \quad u \geq 0,
$$

where

$$
\begin{gathered}
\mathbf{v}(u):=\boldsymbol{\Gamma}(u)+\mathcal{T}_{\mathbf{R}}(\boldsymbol{\alpha}-\mathbf{R}) \boldsymbol{\Gamma}(u), \quad u \geq 0, \\
\boldsymbol{\Gamma}(u):=\frac{1}{c} \int_{u}^{\infty} e^{-\boldsymbol{\alpha}(x-u)} \mathbf{l}(x, u) \mathrm{d} x, \quad u \geq 0, \\
\mathbf{f}(y):=\frac{1}{c} \mathcal{T}_{\mathbf{R}}\left(\boldsymbol{\Lambda}^{1} \circ \mathbf{p}\right)(y), \quad y \geq 0,
\end{gathered}
$$

and $\boldsymbol{\alpha}:=\left(\delta \mathbf{I}-\boldsymbol{\Lambda}^{0}\right) / c$. The general solution of $\mathbf{Z}$ is given by

$$
\mathbf{Z}(u)=\mathbf{v}(u)+\int_{0}^{u} \mathbf{g}(u-y) \mathbf{v}(y) \mathrm{d} y, \quad u \geq 0,
$$

where

$$
\mathbf{g}(y):=\sum_{n=1}^{\infty} \mathbf{f}^{* n}(y), \quad y \geq 0
$$

Proof. With the definitions $\boldsymbol{\alpha}=\left(\delta \mathbf{I}-\boldsymbol{\Lambda}^{0}\right) / c$ and (3.11), by a change of variable (3.4) can be rewritten as

$$
\mathbf{Z}(u)=\frac{1}{c} \mathcal{T}_{\boldsymbol{\alpha}}\left\{\left(\boldsymbol{\Lambda}^{1} \circ \mathbf{p}\right) * \mathbf{Z}\right\}(u)+\boldsymbol{\Gamma}(u) .
$$

For any arbitrary $m \times m$ matrix $\mathbf{S}$, we proceed by pre-multiplying both sides of (3.14) by $\boldsymbol{\alpha}-\mathbf{S}$ and then taking Laplace transform (with argument $\mathbf{S}$ ). This yields

$$
\begin{aligned}
\mathcal{L}\{(\boldsymbol{\alpha}-\mathbf{S}) \mathbf{Z}\}(\mathbf{S}) & =\frac{1}{c} \mathcal{L}\left\{(\boldsymbol{\alpha}-\mathbf{S}) \mathcal{T}_{\boldsymbol{\alpha}}\left\{\left(\boldsymbol{\Lambda}^{1} \circ \mathbf{p}\right) * \mathbf{Z}\right\}\right\}(\mathbf{S})+\mathcal{L}\{(\boldsymbol{\alpha}-\mathbf{S}) \boldsymbol{\Gamma}\}(\mathbf{S}) \\
& =\frac{1}{c}\left[\mathcal{L}\left\{\left(\boldsymbol{\Lambda}^{1} \circ \mathbf{p}\right) * \mathbf{Z}\right\}(\mathbf{S})-\mathcal{L}\left\{\left(\boldsymbol{\Lambda}^{1} \circ \mathbf{p}\right) * \mathbf{Z}\right\}(\boldsymbol{\alpha})\right]+\mathcal{L}\{(\boldsymbol{\alpha}-\mathbf{S}) \boldsymbol{\Gamma}\}(\mathbf{S}) \\
& =\frac{1}{c}\left[\mathcal{L}\left\{\mathcal{L}\left\{\boldsymbol{\Lambda}^{1} \circ \mathbf{p}\right\}(\mathbf{S}) \mathbf{Z}\right\}(\mathbf{S})-\mathcal{L}\left\{\left(\boldsymbol{\Lambda}^{1} \circ \mathbf{p}\right) * \mathbf{Z}\right\}(\boldsymbol{\alpha})\right]+\mathcal{L}\{(\boldsymbol{\alpha}-\mathbf{S}) \boldsymbol{\Gamma}\}(\mathbf{S}),
\end{aligned}
$$


where Corollaries 1 and 2 have been applied in the second and third equalities. By rearranging terms, we arrive at

$$
\mathcal{L}\left\{\left[(\boldsymbol{\alpha}-\mathbf{S})-\frac{1}{c} \mathcal{L}\left\{\boldsymbol{\Lambda}^{1} \circ \mathbf{p}\right\}(\mathbf{S})\right] \mathbf{Z}\right\}(\mathbf{S})=-\frac{1}{c} \mathcal{L}\left\{\left(\boldsymbol{\Lambda}^{1} \circ \mathbf{p}\right) * \mathbf{Z}\right\}(\boldsymbol{\alpha})+\mathcal{L}\{(\boldsymbol{\alpha}-\mathbf{S}) \boldsymbol{\Gamma}\}(\mathbf{S}) .
$$

It is instructive to note that the term inside the square bracket on the left-hand side is simply a constant, and the Laplace transform is taken with respect to the argument of the function $\mathbf{Z}$ only. Because $\mathbf{R}$ satisfies (3.7) (and it is assumed that $\mathbf{Z}\left(\rho_{i}\right)$ is finite for $i=1,2, \ldots, m$ ), substitution of $\mathbf{S}=\mathbf{R}$ into the above equation yields

$$
\frac{1}{c} \mathcal{L}\left\{\left(\boldsymbol{\Lambda}^{1} \circ \mathbf{p}\right) * \mathbf{Z}\right\}(\boldsymbol{\alpha})=\mathcal{L}\{(\boldsymbol{\alpha}-\mathbf{R}) \boldsymbol{\Gamma}\}(\mathbf{R}) .
$$

Back substitution of (3.16) into (3.15) leads to

$$
\mathcal{L}\left\{\left[(\boldsymbol{\alpha}-\mathbf{S})-\frac{1}{c} \mathcal{L}\left\{\boldsymbol{\Lambda}^{1} \circ \mathbf{p}\right\}(\mathbf{S})\right] \mathbf{Z}\right\}(\mathbf{S})=-\mathcal{L}\{(\boldsymbol{\alpha}-\mathbf{R}) \boldsymbol{\Gamma}\}(\mathbf{R})+\mathcal{L}\{(\boldsymbol{\alpha}-\mathbf{S}) \boldsymbol{\Gamma}\}(\mathbf{S}) .
$$

From now on it is sufficient to consider $\mathbf{S}$ in the form of $s \mathbf{I}$ where $s \geq 0$ is a scalar constant. Then the above equation reduces to

$$
\mathcal{L}\left\{\left[(\boldsymbol{\alpha}-s \mathbf{I})-\frac{1}{c} \mathcal{L}\left\{\boldsymbol{\Lambda}^{1} \circ \mathbf{p}\right\}(s \mathbf{I})\right] \mathbf{Z}\right\}(s \mathbf{I})=-\mathcal{L}\{(\boldsymbol{\alpha}-\mathbf{R}) \boldsymbol{\Gamma}\}(\mathbf{R})+\mathcal{L}\{(\boldsymbol{\alpha}-s \mathbf{I}) \boldsymbol{\Gamma}\}(s \mathbf{I}) .
$$

For the moment we focus on the square bracket on the left-hand side. The fact that $\mathbf{R}$ satisfies (3.7) along with the use of Corollary 1 implies

$$
\begin{aligned}
(\boldsymbol{\alpha}-s \mathbf{I})-\frac{1}{c} \mathcal{L}\left\{\boldsymbol{\Lambda}^{1} \circ \mathbf{p}\right\}(s \mathbf{I}) & =\mathbf{R}+\frac{1}{c} \mathcal{L}\left\{\boldsymbol{\Lambda}^{1} \circ \mathbf{p}\right\}(\mathbf{R})-s \mathbf{I}-\frac{1}{c} \mathcal{L}\left\{\boldsymbol{\Lambda}^{1} \circ \mathbf{p}\right\}(s \mathbf{I}) \\
& =\mathbf{R}-s \mathbf{I}-\frac{1}{c} \mathcal{L}\left\{(\mathbf{R}-s \mathbf{I}) \mathcal{T}_{\mathbf{R}}\left(\boldsymbol{\Lambda}^{1} \circ \mathbf{p}\right)\right\}(s \mathbf{I}) \\
& =\mathbf{R}-s \mathbf{I}-\frac{1}{c}(\mathbf{R}-s \mathbf{I}) \mathcal{L}\left\{\mathcal{T}_{\mathbf{R}}\left(\boldsymbol{\Lambda}^{1} \circ \mathbf{p}\right)\right\}(s \mathbf{I}) \\
& =(\mathbf{R}-s \mathbf{I})\left[\mathbf{I}-\frac{1}{c} \mathcal{L}\left\{\mathcal{T}_{\mathbf{R}}\left(\boldsymbol{\Lambda}^{1} \circ \mathbf{p}\right)\right\}(s)\right] .
\end{aligned}
$$

Note that the second last equality follows because $\mathbf{R}$ and $s \mathbf{I}$ commute. Further using this fact again, the left-hand side of (3.17) can be rewritten as

$$
\begin{aligned}
\mathcal{L}\left\{\left[(\boldsymbol{\alpha}-s \mathbf{I})-\frac{1}{c} \mathcal{L}\left\{\boldsymbol{\Lambda}^{1} \circ \mathbf{p}\right\}(s \mathbf{I})\right] \mathbf{Z}\right\}(s \mathbf{I}) & =\mathcal{L}\left\{(\mathbf{R}-s \mathbf{I})\left[\mathbf{I}-\frac{1}{c} \mathcal{L}\left\{\mathcal{T}_{\mathbf{R}}\left(\boldsymbol{\Lambda}^{1} \circ \mathbf{p}\right)\right\}(s)\right] \mathbf{Z}\right\}(s \mathbf{I}) \\
& =(\mathbf{R}-s \mathbf{I})\left[\mathbf{I}-\frac{1}{c} \mathcal{L}\left\{\mathcal{T}_{\mathbf{R}}\left(\boldsymbol{\Lambda}^{1} \circ \mathbf{p}\right)\right\}(s)\right] \mathcal{L}\{\mathbf{Z}\}(s) .
\end{aligned}
$$

On the other hand, the right-hand side of (3.17) equals

$$
\begin{aligned}
-\mathcal{L}\{(\boldsymbol{\alpha}-\mathbf{R}) \boldsymbol{\Gamma}\}(\mathbf{R})+\mathcal{L}\{(\boldsymbol{\alpha}-s \mathbf{I}) \boldsymbol{\Gamma}\}(s \mathbf{I}) & =-\mathcal{L}\{(\boldsymbol{\alpha}-\mathbf{R}) \boldsymbol{\Gamma}\}(\mathbf{R})+\mathcal{L}\{(\boldsymbol{\alpha}-\mathbf{R}) \boldsymbol{\Gamma}\}(s \mathbf{I})+\mathcal{L}\{(\mathbf{R}-s \mathbf{I}) \boldsymbol{\Gamma}\}(s \mathbf{I}) \\
& =\mathcal{L}\left\{(\mathbf{R}-s \mathbf{I}) \mathcal{T}_{\mathbf{R}}(\boldsymbol{\alpha}-\mathbf{R}) \boldsymbol{\Gamma}\right\}(s \mathbf{I})+\mathcal{L}\{(\mathbf{R}-s \mathbf{I}) \boldsymbol{\Gamma}\}(s \mathbf{I}) \\
& =(\mathbf{R}-s \mathbf{I})\left[\mathcal{L}\left\{\mathcal{T}_{\mathbf{R}}(\boldsymbol{\alpha}-\mathbf{R}) \boldsymbol{\Gamma}\right\}(s)+\mathcal{L}\{\boldsymbol{\Gamma}\}(s)\right]
\end{aligned}
$$


where Corollary 1 has been applied in the second equality and the last line again follows from the fact that $\mathbf{R}$ and $s \mathbf{I}$ commute. With the above two equations, (3.17) reduces to

$$
\left[\mathbf{I}-\frac{1}{c} \mathcal{L}\left\{\mathcal{T}_{\mathbf{R}}\left(\boldsymbol{\Lambda}^{1} \circ \mathbf{p}\right)\right\}(s)\right] \mathcal{L}\{\mathbf{Z}\}(s)=\mathcal{L}\left\{\mathcal{T}_{\mathbf{R}}(\boldsymbol{\alpha}-\mathbf{R}) \boldsymbol{\Gamma}\right\}(s)+\mathcal{L}\{\boldsymbol{\Gamma}\}(s),
$$

or equivalently,

$$
\mathcal{L}\{\mathbf{Z}\}(s)=\frac{1}{c} \mathcal{L}\left\{\mathcal{T}_{\mathbf{R}}\left(\boldsymbol{\Lambda}^{1} \circ \mathbf{p}\right)\right\}(s) \mathcal{L}\{\mathbf{Z}\}(s)+\mathcal{L}\{\boldsymbol{\Gamma}\}(s)+\mathcal{L}\left\{\mathcal{T}_{\mathbf{R}}(\boldsymbol{\alpha}-\mathbf{R}) \boldsymbol{\Gamma}\right\}(s)
$$

Inversion of Laplace transforms with respect to $s$ yields

$$
\mathbf{Z}(u)=\frac{1}{c}\left(\left\{\mathcal{T}_{\mathbf{R}}\left(\boldsymbol{\Lambda}^{1} \circ \mathbf{p}\right)\right\} * \mathbf{Z}\right)(u)+\boldsymbol{\Gamma}(u)+\mathcal{T}_{\mathbf{R}}(\boldsymbol{\alpha}-\mathbf{R}) \boldsymbol{\Gamma}(u)
$$

which is the Markov renewal equation (3.9) under the definitions (3.10) and (3.12). The solution (3.13) of (3.9) then follows from Çinlar (1969, Section 3a) or Asmussen (2003, Section VII.4).

Remark 2 If the extended cost function $\mathbf{l}(\cdot, \cdot)$ does not depend on its second argument, $\mathbf{Z}$ reduces to $\mathbf{H}_{1}$ and then (3.11) can be conveniently expressed in terms of the Dickson-Hipp operator as

$$
\boldsymbol{\Gamma}(u)=\frac{1}{c} \int_{u}^{\infty} e^{-\boldsymbol{\alpha}(x-u)} \mathbf{l}(x) \mathrm{d} x=\frac{1}{c} \mathcal{T}_{\boldsymbol{\alpha}} \mathbf{l}(u)
$$

and hence by Lemma $1 \mathbf{v}$ in (3.10) simplifies to give

$$
\mathbf{v}(u)=\frac{1}{c}\left[\mathcal{T}_{\boldsymbol{\alpha}} \mathbf{l}(u)+\mathcal{T}_{\mathbf{R}}(\boldsymbol{\alpha}-\mathbf{R}) \mathcal{T}_{\boldsymbol{\alpha}} \mathbf{l}(u)\right]=\frac{1}{c} \mathcal{T}_{\mathbf{R}} \mathbf{l}(u)
$$

Therefore, the Markov renewal equation (3.9) becomes

$$
\mathbf{H}_{1}(u)=\int_{0}^{u} \mathbf{f}(y) \mathbf{H}_{1}(u-y) \mathrm{d} y+\frac{1}{c} \mathcal{T}_{\mathbf{R}} \mathbf{l}(u), \quad u \geq 0,
$$

which is an extension of Feng (2009b, Theorem 3.1).

Remark 3 Theorem 1 can also be regarded as a generalization of Cheung et al. (2010a, Theorem 2.1) which considered the scalar version of the Gerber-Shiu function $\boldsymbol{\Phi}$ in the classical compound Poisson risk model. It is not difficult to see that their result can be retrieved from ours under the choice of cost function in Proposition 7.

Remark 4 In what follows we shall demonstrate that the matrix $\int_{0}^{\infty} \mathbf{f}(y) \mathrm{d} y$ (with $\mathbf{f}$ defined by (3.12)) is strictly substochastic. By letting $w(\cdot, \cdot) \equiv 1$ in Remark 1 , one observes that $\mathbb{E}^{(u, i)}\left[e^{-\delta \tau} I(\tau<\right.$ $\infty)$ ] is a special case of $C_{1, i}(u)$ under $\varpi_{k j}(x, y)=I(y<0)$. This $C_{1, i}$ is in turn a special case of $H_{1, i}$ according to Proposition 1(1) (with $n=1$ ) with cost function

$$
\mathbf{l}(x)=\int_{x}^{\infty}\left(\Lambda^{1} \circ \mathbf{p}\right)(y) \mathbf{1} \mathrm{d} y=\int_{0}^{\infty}\left(\Lambda^{1} \circ \mathbf{p}\right)(x+y) \mathrm{d} y \mathbf{1} .
$$


Hence, by putting $u=0$ into (3.19), we arrive at

$$
\begin{aligned}
\mathbb{E}^{0}\left[e^{-\delta \tau} I(\tau<\infty)\right] & =\frac{1}{c} \mathcal{T}_{\mathbf{R}} \mathbf{l}(0)=\frac{1}{c} \int_{0}^{\infty} e^{-\mathbf{R} x} \int_{0}^{\infty}\left(\boldsymbol{\Lambda}^{1} \circ \mathbf{p}\right)(x+y) \mathrm{d} y \mathrm{~d} x \mathbf{1} \\
& =\frac{1}{c} \int_{0}^{\infty} \int_{0}^{\infty} e^{-\mathbf{R} x}\left(\boldsymbol{\Lambda}^{1} \circ \mathbf{p}\right)(x+y) \mathrm{d} x \mathrm{~d} y \mathbf{1}=\frac{1}{c} \int_{0}^{\infty} \mathcal{T}_{\mathbf{R}}\left(\boldsymbol{\Lambda}^{1} \circ \mathbf{p}\right)(y) \mathrm{d} y \mathbf{1} \\
& =\int_{0}^{\infty} \mathbf{f}(y) \mathrm{d} y \mathbf{1},
\end{aligned}
$$

where the last line follows from the definition (3.12). Note that each element of the left-hand side, namely $\mathbb{E}^{(0, i)}\left[e^{-\delta \tau} I(\tau<\infty)\right]$, is strictly less than 1 under either $\delta>0$ or the positive security loading

condition (1.5). Hence, we conclude that $\int_{0}^{\infty} \mathbf{f}(y) \mathrm{d} y \mathbf{1}<\mathbf{1}$, implying that the matrix $\int_{0}^{\infty} \mathbf{f}(y) \mathrm{d} y$ is strictly substochastic. Therefore, the Markov renewal equation (3.9) can be viewed as a matrix form of a defective renewal equation, which also arises in the ruin theory literature (see e.g. Ahn and Badescu (2007) and Cheung and Landriault (2009b)). In addition, the solution is known to be unique as well (see e.g. Miyazawa (2002)). We refer interested readers to e.g. Wu (1999), Miyazawa (2002) and Li and Luo (2005) for two-sided bounds and asymptotics for the solution of a matrix defective renewal equation.

\section{Examples and applications}

\subsection{Solution to $\Phi$}

As an example, we derive the solution of $\boldsymbol{\Phi}$ using Theorem 1. From Proposition $7, \boldsymbol{\Phi}_{\cdot j}$ is a special case of $\mathbf{Z}$ with cost function (3.5), and therefore $\mathbf{\Gamma}$ in (3.11) reduces to

$$
\boldsymbol{\Gamma}(u)=\frac{1}{c} \int_{u}^{\infty} e^{-\boldsymbol{\alpha}(x-u)} \int_{x}^{\infty} w(x, y-x, u)\left(\boldsymbol{\Lambda}^{1} \circ \mathbf{p}(y)\right)_{\cdot j} \mathrm{~d} y \mathrm{~d} x=\boldsymbol{\beta}_{\cdot j}(u),
$$

according to the definition (2.19), i.e. $\boldsymbol{\Gamma}$ is simply the $j$-th column of $\boldsymbol{\beta}$. This implies that $\boldsymbol{\Phi}_{\cdot j}$ satisfies the Markov renewal equation (3.9) with $\mathbf{v}(u)=\boldsymbol{\beta}_{\cdot j}(u)+\mathcal{T}_{\mathbf{R}}(\boldsymbol{\alpha}-\mathbf{R}) \boldsymbol{\beta}_{\cdot j}(u)$. Piecing together all the column vectors, we have that $\boldsymbol{\Phi}$ satisfies

$$
\boldsymbol{\Phi}(u)=\int_{0}^{u} \mathbf{f}(y) \boldsymbol{\Phi}(u-y) \mathrm{d} y+\mathbf{v}_{*}(u), \quad u \geq 0
$$

where

$$
\mathbf{v}_{*}(u):=\boldsymbol{\beta}(u)+\mathcal{T}_{\mathbf{R}}(\boldsymbol{\alpha}-\mathbf{R}) \boldsymbol{\beta}(u), \quad u \geq 0 .
$$

Parallel to (3.13), the solution of $\boldsymbol{\Phi}$ is given by

$$
\boldsymbol{\Phi}(u)=\mathbf{v}_{*}(u)+\int_{0}^{u} \mathbf{g}(u-y) \mathbf{v}_{*}(y) \mathrm{d} y, \quad u \geq 0 .
$$

In the same manner as in Cheung et al. (2010a, Theorem 2.2), we can express the above solution in the form of (2.18) with the function $\chi$ explicitly identified. Substitution of (4.1) into (4.2) gives

$$
\boldsymbol{\Phi}(u)=\boldsymbol{\beta}(u)+\mathcal{T}_{\mathbf{R}}(\boldsymbol{\alpha}-\mathbf{R}) \boldsymbol{\beta}(u)+\int_{0}^{u} \mathbf{g}(u-y) \boldsymbol{\beta}(y) \mathrm{d} y+\int_{0}^{u} \mathbf{g}(u-y) \mathcal{T}_{\mathbf{R}}(\boldsymbol{\alpha}-\mathbf{R}) \boldsymbol{\beta}(y) \mathrm{d} y .
$$


While the second term in the above equation can be represented as

$$
\mathcal{T}_{\mathbf{R}}(\boldsymbol{\alpha}-\mathbf{R}) \boldsymbol{\beta}(u)=\int_{u}^{\infty} e^{-\mathbf{R}(y-u)}(\boldsymbol{\alpha}-\mathbf{R}) \boldsymbol{\beta}(y) \mathrm{d} y,
$$

the last integral equals, by changing the order of integrations,

$$
\begin{aligned}
& \int_{0}^{u} \mathbf{g}(u-y) \mathcal{T}_{\mathbf{R}}(\boldsymbol{\alpha}-\mathbf{R}) \boldsymbol{\beta}(y) \mathrm{d} y=\int_{0}^{u} \mathbf{g}(u-y) \int_{y}^{\infty} e^{-\mathbf{R}(z-y)}(\boldsymbol{\alpha}-\mathbf{R}) \boldsymbol{\beta}(z) \mathrm{d} z \mathrm{~d} y \\
= & \int_{0}^{u}\left(\int_{0}^{z} \mathbf{g}(u-y) e^{-\mathbf{R}(z-y)} \mathrm{d} y\right)(\boldsymbol{\alpha}-\mathbf{R}) \boldsymbol{\beta}(z) \mathrm{d} z+\int_{u}^{\infty}\left(\int_{0}^{u} \mathbf{g}(u-y) e^{-\mathbf{R}(z-y)} \mathrm{d} y\right)(\boldsymbol{\alpha}-\mathbf{R}) \boldsymbol{\beta}(z) \mathrm{d} z .
\end{aligned}
$$

With the above two equations, the solution (4.2) can be written in the form (2.18) with $\chi$ given by

$$
\chi(u, z)= \begin{cases}\mathbf{g}(u-z)+\left(\int_{0}^{z} \mathbf{g}(u-y) e^{-\mathbf{R}(z-y)} \mathrm{d} y\right)(\boldsymbol{\alpha}-\mathbf{R}), & z<u . \\ {\left[e^{-\mathbf{R}(z-u)}+\left(\int_{0}^{u} \mathbf{g}(u-y) e^{-\mathbf{R}(z-y)} \mathrm{d} y\right)\right](\boldsymbol{\alpha}-\mathbf{R}),} & z \geq u .\end{cases}
$$

The above representation for $\boldsymbol{\chi}$ is an extension of Cheung et al. (2010a, Equation (18)) and complements Cheung and Landriault (2009b, Equation (4.24)). We remark that one may also directly

derive $\boldsymbol{\chi}$ with $\boldsymbol{\chi}_{\cdot j}$ being a solution of (3.19) using Proposition 4. This will lead to the same result as in (4.3) and the details are omitted here.

\subsection{Minimum surplus before ruin}

Here we aim at using the results in previous sections to study the Gerber-Shiu function which further involves the minimum surplus level before ruin $\underline{U}_{\tau^{-}}=\inf _{0 \leq s<\tau} U_{s}$ in the penalty function. We consider $\boldsymbol{\Phi}_{\min }(u)$ whose $(i, j)$-th element is given by

$$
\phi_{\min , i j}(u):=\mathbb{E}^{(u, i)}\left[e^{-\delta \tau} w_{\min }\left(U_{\tau^{-}},\left|U_{\tau}\right|, \underline{U}_{\tau^{-}}, R_{N_{\tau}-1}\right) I\left(\tau<\infty, J_{\tau}=j\right)\right], \quad u \geq 0,
$$

where $w_{\min }(\cdot, \cdot, \cdot, \cdot)$ is now a four-variable penalty function. Note that the minimum $\underline{U}_{\tau^{-}}$is placed in the third argument just to be consistent with other papers in the literature (Cheung and Landriault (2009b), Cheung et al. (2010b) and Woo (2010, 2012)).

To analyze $\boldsymbol{\Phi}_{\min }$, for the moment we focus on the Gerber-Shiu function $\boldsymbol{\Phi}$ with representation given by Proposition 5. First, it will be useful to rewrite the contribution by ruin occurring upon the first claim (2.19) as

$$
\boldsymbol{\beta}(u)=\int_{0}^{\infty} \int_{u}^{\infty} w(x, y, u) \mathbf{h}_{1}(x, y \mid u) \mathrm{d} x \mathrm{~d} y, \quad u \geq 0,
$$

where

$$
\mathbf{h}_{1}(x, y \mid u):=\frac{1}{c} e^{\left(-\delta \mathbf{I}+\boldsymbol{\Lambda}^{0}\right)\left(\frac{x-u}{c}\right)}\left(\boldsymbol{\Lambda}^{1} \circ \mathbf{p}(x+y)\right), \quad x>u ; y>0,
$$

is the discounted density of $\left(U_{\tau^{-}},\left|U_{\tau}\right|\right)$ at $(x, y)$ given $U_{0}=u$ for ruin on the first claim (implying $\tau=(x-u) / c$ and $\left.R_{N_{\tau}-1}=u\right)$. Substitution of (4.4) into (2.18) yields

$$
\begin{aligned}
\mathbf{\Phi}(u) & =\int_{0}^{\infty} \int_{u}^{\infty} w(x, y, u) \mathbf{h}_{1}(x, y \mid u) \mathrm{d} x \mathrm{~d} y+\int_{0}^{\infty} \chi(u, z) \int_{0}^{\infty} \int_{z}^{\infty} w(x, y, z) \mathbf{h}_{1}(x, y \mid z) \mathrm{d} x \mathrm{~d} y \mathrm{~d} z \\
& =\int_{0}^{\infty} \int_{u}^{\infty} w(x, y, u) \mathbf{h}_{1}(x, y \mid u) \mathrm{d} x \mathrm{~d} y+\int_{0}^{\infty} \int_{0}^{\infty} \int_{z}^{\infty} w(x, y, z) \mathbf{h}_{2}(x, y, z \mid u) \mathrm{d} x \mathrm{~d} y \mathrm{~d} z, \quad u \geq 0,
\end{aligned}
$$


where

$$
\mathbf{h}_{2}(x, y, z \mid u):=\chi(u, z) \mathbf{h}_{1}(x, y \mid z), \quad x>z \geq 0 ; y>0
$$

clearly represents the discounted density of $\left(U_{\tau^{-}},\left|U_{\tau}\right|, R_{N_{\tau}-1}\right)$ at $(x, y, z)$ given $U_{0}=u$ for ruin on claims subsequent to the first. Using the fact that $\chi(0, z)=e^{-\mathbf{R} z}(\boldsymbol{\alpha}-\mathbf{R})$ (by putting $u=0$ in (4.3)) along with (4.5), it is straightforward to verify by applying Lemma 1 that

$$
\int_{0}^{\infty} \mathbf{h}_{1}(x, y \mid 0) \mathrm{d} x+\int_{0}^{\infty} \int_{z}^{\infty} \chi(0, z) \mathbf{h}_{1}(x, y \mid z) \mathrm{d} x \mathrm{~d} z=\mathbf{f}(y),
$$

where $\mathbf{f}$ is defined by (3.12). Hence $\mathbf{f}(y)$ is the so-called 'ladder height' matrix, i.e. its $(i, j)$-th element $[\mathbf{f}(y)]_{i j}$ represents the density of the amount of first drop (at $y$ ) of the process $\mathcal{U}$ below its initial level together with the event that $\mathcal{J}$ is in state $j$ immediately after the drop, given that $J_{0}=i$.

Now, we can analyze $\Phi_{\min }(u)$ by conditioning on the first drop of $\mathcal{U}$ below the initial level $U_{0}=u$ and keeping track of the environmental states of $\mathcal{J}$. As in Cheung and Landriault (2009b, Equations (2.6) and (2.7)), this results in the matrix defective renewal equation

$$
\boldsymbol{\Phi}_{\min }(u)=\int_{0}^{u} \mathbf{f}(y) \boldsymbol{\Phi}_{\min }(u-y) \mathrm{d} y+\mathbf{v}_{\min }(u), \quad u \geq 0,
$$

where

$$
\begin{aligned}
\mathbf{v}_{\min }(u):= & \int_{u}^{\infty} \int_{0}^{\infty} w_{\min }(x+u, y-u, u, u) \mathbf{h}_{1}(x, y \mid 0) \mathrm{d} x \mathrm{~d} y \\
& +\int_{u}^{\infty} \int_{0}^{\infty} \int_{z}^{\infty} w_{\min }(x+u, y-u, u, z+u) \mathbf{h}_{2}(x, y, z \mid 0) \mathrm{d} x \mathrm{~d} z \mathrm{~d} y, \quad u \geq 0 .
\end{aligned}
$$

Note that all components of the matrix defective renewal equation are already known. Its solution is thus given by (4.2) with $\mathbf{v}_{*}$ replaced by $\mathbf{v}_{\min }$.

\subsection{Matrix scale function and maximum surplus before ruin}

This subsection demonstrates that some of our intermediate results are indeed useful for analyzing the 'scale function' pertaining to the MArP risk model, which in turn allows for the analysis of the maximum surplus before ruin. To this end, we define the first passage time $\tau_{b}^{*}:=\inf \left\{t \geq 0: U_{t}=b\right\}$ and the Laplace transform

$$
\eta_{i j}(u ; b):=\mathbb{E}^{(u, i)}\left[e^{-\delta \tau_{b}^{*}} I\left(\tau_{b}^{*}<\tau, J_{\tau_{b}^{*}}=j\right)\right], \quad 0 \leq u \leq b .
$$

It is known (see e.g. Cheung and Landriault (2010, Equations (9) and (11))) that $\boldsymbol{\eta}(u ; b):=$ $\left[\eta_{i j}(u ; b)\right]_{i, j=1}^{m}$ admits the representation

$$
\boldsymbol{\eta}(u ; b)=\mathbf{Q}(u)[\mathbf{Q}(b)]^{-1}, \quad 0 \leq u \leq b,
$$

where the matrix function $\mathbf{Q}$ is characterized by its Laplace transform as

$$
\mathcal{L}\{\mathbf{Q}\}(s)=\left[\left(s-\frac{\delta}{c}\right) \mathbf{I}+\frac{1}{c} \boldsymbol{\Lambda}^{0}+\frac{1}{c} \mathcal{L}\left\{\boldsymbol{\Lambda}^{1} \circ \mathbf{p}\right\}(s)\right]^{-1}, \quad u \geq 0 .
$$


Note that $\mathbf{Q}$ can be regarded as a matrix version of the so-called scale function in the literature of Lévy processes. However, an expression for $\mathbf{Q}$ has not been given by Cheung and Landriault (2010). It turns out that this is possible with the use of matrix Dickson-Hipp operators. Simple manipulations followed by the use of (3.18) lead to

$$
\begin{aligned}
\mathcal{L}\{\mathbf{Q}\}(s) & =-\left[(\boldsymbol{\alpha}-s \mathbf{I})-\frac{1}{c} \mathcal{L}\left\{\boldsymbol{\Lambda}^{1} \circ \mathbf{p}\right\}(s \mathbf{I})\right]^{-1}=\left\{(s \mathbf{I}-\mathbf{R})\left[\mathbf{I}-\frac{1}{c} \mathcal{L}\left\{\mathcal{T}_{\mathbf{R}}\left(\boldsymbol{\Lambda}^{1} \circ \mathbf{p}\right)\right\}(s)\right]\right\}^{-1} \\
& =\left[\mathbf{I}-\frac{1}{c} \mathcal{L}\left\{\mathcal{T}_{\mathbf{R}}\left(\boldsymbol{\Lambda}^{1} \circ \mathbf{p}\right)\right\}(s)\right]^{-1}(s \mathbf{I}-\mathbf{R})^{-1}=[\mathbf{I}-\mathcal{L}\{\mathbf{f}\}(s)]^{-1}(s \mathbf{I}-\mathbf{R})^{-1},
\end{aligned}
$$

which implies, upon rearrangements,

$$
\mathcal{L}\{\mathbf{Q}\}(s)=\mathcal{L}\{\mathbf{f}\}(s) \mathcal{L}\{\mathbf{Q}\}(s)+(s \mathbf{I}-\mathbf{R})^{-1} .
$$

By inverting the Laplace transforms with respect to $s$, we arrive at the Markov renewal equation

$$
\mathbf{Q}(u)=\int_{0}^{u} \mathbf{f}(y) \mathbf{Q}(u-y) \mathrm{d} y+e^{\mathbf{R} u}, \quad u \geq 0,
$$

which has solution

$$
\mathbf{Q}(u)=e^{\mathbf{R} u}+\int_{0}^{u} \mathbf{g}(u-y) e^{\mathbf{R} y} \mathrm{~d} y, \quad u \geq 0 .
$$

This is a generalization of Cheung and Landriault (2010, Equation (36)) and also the matrix version of Feng and Shimizu (2013a, Equation (4.2)).

With an expression for $\mathbf{Q}$ determined, a direct consequence will be another generalization of Gerber-Shiu function further involving the maximum surplus before ruin $\bar{U}_{\tau^{-}}=\sup _{0 \leq s<\tau} U_{s}$, namely $\Phi_{\max }(u)$, which has $(i, j)$-th element

$$
\phi_{\max , i j}(u):=\mathbb{E}^{(u, i)}\left[e^{-\delta \tau} w\left(U_{\tau^{-}},\left|U_{\tau}\right|\right) w_{*}\left(\bar{U}_{\tau^{-}}\right) I\left(\tau<\infty, J_{\tau}=j\right)\right], \quad u \geq 0 .
$$

From Cheung and Landriault (2010, Equation (19)), one has that

$$
\boldsymbol{\Phi}_{\max }(u)=\int_{u}^{\infty} w_{*}(z) \frac{\mathrm{d}}{\mathrm{d} z}\left[-\boldsymbol{\eta}(u ; z) \mathbf{\Phi}_{C L}(z)\right] \mathrm{d} z=\int_{u}^{\infty} w_{*}(z) \frac{\mathrm{d}}{\mathrm{d} z}\left\{-\mathbf{Q}(u)[\mathbf{Q}(z)]^{-1} \boldsymbol{\Phi}_{C L}(z)\right\} \mathrm{d} z, \quad u \geq 0,
$$

where $\boldsymbol{\Phi}_{C L}(u):=\left[\phi_{C L, i j}(u)\right]_{i, j=1}^{m}$ is the matrix of classical Gerber-Shiu functions defined in Remark 1.

\section{Acknowledgments}

Support for Eric Cheung from the Research Grants Council of the Hong Kong Special Administrative Region (Project Number: HKU 701212P) is gratefully acknowledged. 


\section{A Appendix: The Lundberg's equation and the matrix $\mathbf{R}$}

This Appendix aims at studying the matrix $\mathbf{R}$ which satisfies (3.7) in connection to (3.8) using similar ideas as in Ren (2007) (see also Albrecher and Boxma (2005)). For convenience, let

$$
\mathbf{A}(s):=\left(s-\frac{\delta}{c}\right) \mathbf{I}+\frac{1}{c} \boldsymbol{\Lambda}^{0}+\frac{1}{c} \mathcal{L}\left\{\boldsymbol{\Lambda}^{1} \circ \mathbf{p}\right\}(s) .
$$

Then (3.8) can be represented as

$$
\operatorname{det}[\mathbf{A}(\xi)]=0
$$

which has $m$ roots $\left\{\rho_{i}\right\}_{i=1}^{m}$ with non-negative real parts (e.g. Badescu (2008)) and these roots are assumed to be distinct. This implies that for each fixed $i=1,2, \ldots, m$, there is a non-trivial solution $\boldsymbol{\theta}_{i}:=\left(\theta_{i 1}, \theta_{i 2}, \ldots, \theta_{i m}\right)^{\top}$ to the equation

$$
\left[\mathbf{A}\left(\rho_{i}\right)\right]^{\top} \boldsymbol{\theta}_{i}=\mathbf{0} .
$$

Equivalently, we may say that $\boldsymbol{\theta}_{i}^{\top}$ is the left eigenvector of $\mathbf{A}\left(\rho_{i}\right)$ corresponding to the eigenvalue 0 , so that for $i=1,2, \ldots, m$,

$$
\begin{aligned}
\mathbf{0} & =\boldsymbol{\theta}_{i}^{\top} \mathbf{A}\left(\rho_{i}\right)=\boldsymbol{\theta}_{i}^{\top}\left[\left(\rho_{i}-\frac{\delta}{c}\right) \mathbf{I}+\frac{1}{c} \boldsymbol{\Lambda}^{0}+\frac{1}{c} \mathcal{L}\left\{\boldsymbol{\Lambda}^{1} \circ \mathbf{p}\right\}\left(\rho_{i}\right)\right] \\
& =\rho_{i} \boldsymbol{\theta}_{i}^{\top}+\frac{1}{c} \boldsymbol{\theta}_{i}^{\top}\left(\boldsymbol{\Lambda}^{0}-\delta \mathbf{I}\right)+\frac{1}{c} \int_{0}^{\infty} e^{-\rho_{i} x} \boldsymbol{\theta}_{i}^{\top}\left(\boldsymbol{\Lambda}^{1} \circ \mathbf{p}(x)\right) \mathrm{d} x .
\end{aligned}
$$

Combining the above equations for all $i$, we arrive at

$$
\mathbf{0}=\varrho \boldsymbol{\Theta}+\frac{1}{c} \boldsymbol{\Theta}\left(\boldsymbol{\Lambda}^{0}-\delta \mathbf{I}\right)+\frac{1}{c} \int_{0}^{\infty} e^{-\varrho x} \boldsymbol{\Theta}\left(\boldsymbol{\Lambda}^{1} \circ \mathbf{p}(x)\right) \mathrm{d} x,
$$

where $\boldsymbol{\varrho}:=\operatorname{diag}\left\{\rho_{1}, \rho_{2}, \ldots, \rho_{m}\right\}$ and $\boldsymbol{\Theta}:=\left[\theta_{i j}\right]_{i, j=1}^{m}=\left(\boldsymbol{\theta}_{1}, \boldsymbol{\theta}_{2}, \ldots, \boldsymbol{\theta}_{m}\right)^{\top}$. Pre-multiplying the above equation by $\Theta^{-1}$ leads to

$$
\mathbf{0}=\boldsymbol{\Theta}^{-1} \varrho \boldsymbol{\Theta}+\frac{1}{c}\left(\boldsymbol{\Lambda}^{0}-\delta \mathbf{I}\right)+\frac{1}{c} \int_{0}^{\infty} \boldsymbol{\Theta}^{-1} e^{-\varrho x} \boldsymbol{\Theta}\left(\boldsymbol{\Lambda}^{1} \circ \mathbf{p}(x)\right) \mathrm{d} x .
$$

Let $\mathbf{R}:=\Theta^{-1} \varrho \Theta$. Then we have the well-known property regarding matrix exponentials that $e^{\mathbf{R} y}=\boldsymbol{\Theta}^{-1} e^{\varrho y} \boldsymbol{\Theta}$ and hence $e^{\varrho y}=\boldsymbol{\Theta} e^{\mathbf{R} y} \boldsymbol{\Theta}^{-1}$. Thus, (A.1) can be rewritten as

$$
\mathbf{0}=\mathbf{R}+\frac{1}{c}\left(\boldsymbol{\Lambda}^{0}-\delta \mathbf{I}\right)+\frac{1}{c} \int_{0}^{\infty} e^{-\mathbf{R} x}\left(\boldsymbol{\Lambda}^{1} \circ \mathbf{p}(x)\right) \mathrm{d} x,
$$

which is equivalent to (3.7). In other words, $\mathbf{R}$ satisfying (3.7) can be evaluated by $\mathbf{R}=\boldsymbol{\Theta}^{-1} \boldsymbol{\varrho} \Theta$.

\section{References}

[1] Ahn, S. And Badescu, A.L. 2007. On the analysis of the Gerber-Shiu discounted penalty function for risk processes with Markovian arrivals. Insurance: Mathematics and Economics 41(2): 234-249. 
[2] Ahn, S., Badescu, A.L. and Ramaswami, V. 2007. Time dependent analysis of finite buffer fluid flows and risk models with a dividend barrier. Queueing Systems 55(4): 207-222.

[3] Albrecher, H. And Boxma, O.J. 2005. On the discounted penalty function in a Markovdependent risk model. Insurance: Mathematics and Economics 37(3): 650-672.

[4] Asmussen, S. 2003. Applied Probability and Queues. 2nd Edition. New York: Springer.

[5] Badescu, A.L. 2008. Discussion of 'The discounted joint distribution of the surplus prior to ruin and the deficit at ruin in a Sparre Andersen model.' North American Actuarial Journal $12(2): 210-212$.

[6] Badescu, A.L., Breuer, L., Da Silva Soares, A., Latouche, G., Remiche, M.-A. And Stanford, D. 2005. Risk processes analyzed as fluid queues. Scandinavian Actuarial Journal 2005(2): 127-141.

[7] Badescu, A.L., Drekic, S. and Landriault, D. 2007a. Analysis of a threshold dividend strategy for a MAP risk model. Scandinavian Actuarial Journal 2007(4): 227-247.

[8] Badescu, A.L., Drekic, S. And Landriault, D. 2007b. On the analysis of a multi-threshold Markovian risk model. Scandinavian Actuarial Journal 2007(4): 248-260.

[9] Badescu, A.L. And Landriault, D. 2008. Recursive calculation of the dividend moments in a multi-threshold risk model. North American Actuarial Journal 12(1): 74-88.

[10] Cai, J., Feng, R. And Willmot, G.E. 2009. On the total discounted operating costs up to default and its applications. Advances in Applied Probability 41(2): 495-522.

[11] Cheung, E.C.K. 2007. Discussion of 'Moments of the dividend payments and related problems in a Markov-modulated risk model'. North American Actuarial Journal 11(4): 145-148.

[12] Cheung, E.C.K. 2011. A generalized penalty function in Sparre Andersen risk models with surplus-dependent premium. Insurance: Mathematics and Economics 48(3): 383-397.

[13] Cheung, E.C.K. And Landriault, D. 2009a. Perturbed MAP risk models with dividend barrier strategies. Journal of Applied Probability 46(2): 521-541.

[14] Cheung, E.C.K. And Landriault, D. 2009b. Analysis of a generalized penalty function in a semi-Markovian risk model. North American Actuarial Journal 13(4): 497-513.

[15] Cheung, E.C.K. And Landriault, D. 2010. A generalized penalty function with the maximum surplus prior to ruin in a MAP risk model. Insurance: Mathematics and Economics 46(1): 127-134.

[16] Cheung, E.C.K., Landriault, D., Willmot, G.E. and Woo, J.-K. 2010a. Gerber-Shiu analysis with a generalized penalty function. Scandinavian Actuarial Journal 2010(3): 185-199.

[17] Cheung, E.C.K., Landriault, D., Willmot, G.E. And Woo, J.-K. 2010b. Structural properties of Gerber-Shiu functions in dependent Sparre Andersen models. Insurance: Mathematics and Economics 46(1): 117-126. 
[18] Cheung, E.C.K., Landriault, D., Willmot, G.E. and Woo, J.-K. 2011. On orderings and bounds in a generalized Sparre Andersen risk model. Applied Stochastic Models in Business and Industry 27(1): 51-60.

[19] ÇInlar, E. 1969. Markov renewal theory. Advances in Applied Probability 1(2): 123-187.

[20] Dickson, D.C.M. And Hipp, C. 2001. On the time to ruin for Erlang(2) risk processes. Insurance: Mathematics and Economics 29(3): 333-344.

[21] FEnG, R. 2009a. On the total operating costs up to default in a renewal risk model. Insurance: Mathematics and Economics 45(2): 305-314.

[22] FEnG, R. 2009b. A matrix operator approach to the analysis of ruin-related quantities in the phase-type renewal risk model. Schweizerische Aktuarvereinigung Mitteilungen 2009(1\&2): 7187. Correction available at http://www.math.uiuc.edu/ rfeng.

[23] Feng, R. 2011. An operator-based approach to the analysis of ruin-related quantities in jump diffusion risk models. Insurance: Mathematics and Economics 48(2): 304-313.

[24] Feng, R. And Shimizu, Y. 2013a. On a generalization from ruin to default in Lévy insurance risk model. Methodology and Computing in Applied Probability. In press.

[25] Feng, R. And Shimizu, Y. 2013b. Potential measure of spectrally negative Markov additive process with applications to ruin theory. Preprint.

[26] Gerber, H.U. And Shiu, E.S.W. 1998. On the time value of ruin. North American Actuarial Journal 2(1): 48-72.

[27] Karatzas, I. And Shreve, S.E. 1991. Brownian Motion and Stochastic Calculus. 2nd Edition. New York: Springer.

[28] Latouche, G. And Ramaswami, V. 1999. Introduction to Matrix Analytic Methods in Stochastic Modeling. Philadelphia: ASA SIAM.

[29] Li, S. AND Lu, Y. 2007. Moments of the dividend payments and related problems in a Markovmodulated risk model. North American Actuarial Journal 11(2): 65-76.

[30] Li, S. AND LU, Y. 2008. The decompositions of the discounted penalty functions and dividendspenalty identity in a Markov-modulated risk model. ASTIN Bulletin 38(1): 53-71.

[31] Li, G. AND Luo, J. 2005. Upper and lower bounds for the solutions of Markov renewal equations. Mathematical Methods of Operations Research 62(2): 243-253.

[32] Miyazawa, M. 2002. A Markov renewal approach to the asymptotic decay of the tail probabilities in risk and queueing processes. Probability in the Engineering and Informational Sciences 16(2): 139-150.

[33] Neuts, M.F. 1989. Structured Stochastic Matrices of $M / G / 1$ Type and Their Applications. New York: Marcel Dekker. 
[34] REN, J. 2007. The discounted joint distribution of the surplus prior to ruin and the deficit at ruin in a Sparre Andersen model. North American Actuarial Journal 11(3): 128-136.

[35] Woo, J.-K. 2010. Some remarks on delayed renewal risk models. ASTIN Bulletin 40(1): 199219.

[36] Woo, J.-K. 2012. A generalized penalty function for a class of discrete renewal processes. Scandinavian Actuarial Journal 2012(2): 130-152.

[37] Wu, Y. 1999. Bounds for the ruin probability under a markovian modulated risk model. Communications in Statistics - Stochastic Models 15(1): 125-136.

[38] ZHU, J. AND YANG, H. 2008. Ruin theory for a Markov regime-switching model under a threshold dividend strategy. Insurance: Mathematics and Economics 42(1): 311-318.

[39] Zhu, J. AND YANG, H. 2009. On differentiability of ruin functions under Markov-modulated models. Stochastic Processes and their Applications 119(5): 1673-1695. 